Supporting Information

https://doi.org/10.1021/acs.jpca.1c04081

\title{
Evidence of IR-Induced Chemistry in a Neat Solid: Tautomerization of Thiotropolone by Thermal, Electronic, and Vibrational Excitations
}

Nelson A. M. Pereira, ${ }^{1}$ Cláudio M. Nunes, ${ }^{1, *}$ Igor Reva, ${ }^{1,2}$ and Rui Fausto ${ }^{1}$

${ }^{1}$ University of Coimbra, CQC, Department of Chemistry, 3004-535 Coimbra, Portugal
${ }^{2}$ University of Coimbra, CIEPQPF, Department of Chemical Engineering, 3030-790 Coimbra, Portugal
Email: npereira@qui.uc.pt, cmnunes@qui.uc.pt, reva@eq.uc.pt, rfausto@ci.uc.pt

Table of contents

1. Vibrational assignment of thiotropolone in Ar Matrix S2

2. Figures S5

3. Tables $\quad$ S15

4. References $\quad$ S39 


\section{Vibrational assignment of s-OH and a-SH in argon matrix:}

The irradiation $(\lambda \geq 400 \mathrm{~nm}$ ) of the thione-enol s-OH form deposited in an argon matrix at 15 $\mathrm{K}$ was found to produce a new tautomer ascribed as the thiol-keto a-SH form. Thus, the IR spectra of the sample before and after irradiation contain signatures of two isomeric forms of TT: exclusively the s-OH tautomer before any irradiation (Figure S5a), and essentially the a-SH tautomer after irradiation (Figure S5b). This permits to extract the individual spectrum of the a-SH tautomer (Figure S5c) and carry out the vibrational assignment of the two isomers, which is presented in Figure S6. The first striking difference between the experimental spectra of the $\mathbf{s - O H}$ and a-SH tautomers, is a much higher total absorption intensity of the s-OH form, as compared to the a-SH form. Note that experimental spectra in frames (b) and (c) are shown in the same scale, and they were obtained from the same sample and represent equimolar amounts. The total measured experimental intensity of all bands in the fingerprint (1700-450 $\mathrm{cm}^{-1}$ ) range for $(\mathbf{s}-\mathbf{O H})$ is 1.547 times higher than that for $(\mathbf{a}-\mathbf{S H})$. In order to verify this experimental observation, the $(\mathbf{s}-\mathbf{O H}) /(\mathbf{a}-\mathbf{S H})$ ratio of the total computed IR intensities in the same range was assessed. This computed ratio equals to 1.569, being in excellent match with the experimental value. Besides a very good overall correspondence between the computed and experimental spectra of each individual TT isomer, it appears instructive to distinguish several particular bands / spectral ranges that can be used as discriminative features in the subsequent analysis.

The vibrational assignment of the s-OH form is presented in Tables S2 and S3. The most intense IR absorption of the $\mathbf{s - O H}$ form corresponds to the group of bands in the $1500-1350 \mathrm{~cm}^{-1}$ range (Figure S6a and S6b). These bands correspond to the $v_{10}, v_{11}, v_{12}$, fundamental modes with computed IR intensities from 85 to $232 \mathrm{~km} \mathrm{~mol}^{-1}$, whose sum corresponds to $30 \%$ of intensity in the overall IR spectrum of the $\mathbf{s - O H}$ isomer. The $v_{10}-v_{12}$ fundamental modes correspond to $v(\mathrm{C}=\mathrm{C})$ stretching and $\delta(\mathrm{CH})$ in-plane bending vibrations coupled in different fashions with the $\delta \mathrm{COH}$ mode (Table S2). The experimental spectrum in this range exhibits five strong absorptions, more than the number of predicted fundamentals, and the additional bands must be caused by combination modes which include $v_{21}+v_{23}$, $v_{31}+v_{35}, v_{32}+v_{33}, v_{32}+v_{34}, 2 \times v_{32}$ pairs, all with IR intensities between 12 and $54 \mathrm{~km} \mathrm{~mol}^{-1}$, as predicted by anharmonic vibrational computations (Table S3). Fermi-resonance interactions between the $v_{10}-v_{12}$ fundamentals and the above combinations, resulting in exchange of IR intensities cannot be excluded either. The important message is that the $\mathbf{s - O H}$ form exhibits several intense IR absorptions in the $1500-1350 \mathrm{~cm}^{-1}$ range, and a comparatively weak 
absorption in the 1700-1500 $\mathrm{cm}^{-1}$ range. In the latter range, above $1500 \mathrm{~cm}^{-1}$, the $v_{7}$ and $v_{8}$ fundamental $v(\mathrm{C}=\mathrm{C})$ stretching modes are predicted, which however do not couple with the $\delta \mathrm{COH}$ coordinate (Table S4), and have relatively weak IR absorptions, both computed and observed (Figure S6a and S6b).

Upon the thione-enol $\rightarrow$ thiol-keto tautomerization in TT, there is a dramatic redistribution of spectral intensity above and below $1500 \mathrm{~cm}^{-1}$ (see vertical dotted line in Figure S6 between panels S6b and S6c). The fundamental mode $v_{8}$ of a-SH, a multiplet band between 1605 and $1585 \mathrm{~cm}^{-1}$ (Figure S6c), with predicted intensity of $249 \mathrm{~km} \mathrm{~mol}^{-1}$ (Figure S6d), is alone responsible for $30 \%$ of the overall IR intensity of this form, and when summed up with $v_{7}$ and $v_{9}$ this share increases over $42 \%$. Indeed, in the experimental spectrum of a-SH, the integrated intensity over the $1700-1500 \mathrm{~cm}^{-1}$ range corresponds to $50 \%$ (Figure S6c). The reason behind these dramatic changes is that the thiol form of TT acquires the carbonyl $\mathrm{C}=\mathrm{O}$ group, a dipole strongly absorbing in infrared. Indeed, the vibrational analysis of a-SH shows that the $v_{8}$ fundamental mode is mainly due to the $v(C=O)$ stretching (Table $\mathrm{S} 4)$, and the $v_{7}$ mode due to $v(\mathrm{C}=\mathrm{C})$ is also coupled with $v(\mathrm{C}=\mathrm{O})$. The $v_{10}-v_{12}$ fundamental modes of a-SH, due to the $v(\mathrm{C}=\mathrm{C})$ stretching and $\delta(\mathrm{CH})$ in-plane bending vibrations, appearing below $1500 \mathrm{~cm}^{-1}$, are not coupled anymore with the $\delta \mathrm{COH}$ coordinate (because the $\mathrm{COH}$ group is absent in a-SH), nor with $v(\mathrm{C}=\mathrm{O})$, and, hence, these modes become weak in infrared, as evidenced by both the experimental and computed spectra (Figure S6c and S6d). The multiplet character of the experimental absorption of a-SH above $1500 \mathrm{~cm}^{-1}$ must be caused by combination modes which include $v_{21}+v_{22}, v_{17}+v_{24}, v_{14}+v_{26}, v_{30}+v_{33}$ pairs, all with IR intensities above $10 \mathrm{~km} \mathrm{~mol}^{-1}$, as predicted by anharmonic vibrational computations. All these combination bands have predicted frequencies in the vicinity of $v_{7}-v_{9}$ fundamentals (Table S5).

Both TT isomers contain five ring $\mathrm{CH}$ groups. These $\mathrm{CH}$ groups give rise to five $\gamma(\mathrm{CH})$ out-of-plane vibrations, where typically all $\mathrm{CH}$ groups contribute in different phases (modes from $\gamma_{\mathrm{a}}(\mathrm{CH})$ to $\gamma_{\mathrm{e}}(\mathrm{CH})$ in Tables S3 and S5). Depending on the phase of each $\mathrm{CH}$ group, the resulting change of the dipole moment is either cancelled or enhanced. When displacements of all of these $\mathrm{CH}$ groups are coupled in the same phase, this typically gives rise to a very strong infrared band. For example, in the structurally similar phenol monomer, one of five $\gamma(\mathrm{CH})$ modes originates one of the strongest IR bands observed at $752 \mathrm{~cm}^{-1}$, with computed IR intensity of $84.9 \mathrm{~km} \mathrm{~mol}^{-1}$. ${ }^{1}$ In the $\mathbf{s}-\mathbf{O H}$ form of TT, it is the $\gamma_{\mathrm{e}}(\mathrm{CH})$ mode $\left(v_{33}\right.$, see Figure S6b), which is predicted to have an IR intensity of $71.8 \mathrm{~km} \mathrm{~mol}^{-1}$, and is observed as a 
strong band at $734.8 / 732.6 \mathrm{~cm}^{-1}$. The frequencies and couplings of the $\gamma(\mathrm{CH})$ vibrations sensitively depend on the geometry of the nearest groups, which in TT correspond to the OCCS fragment, with an $\mathrm{H}$ atom located on either oxygen (in $\mathbf{s - O H}$ ) or sulphur (in $\mathbf{a - S H}$ ). Indeed, upon thione-enol $\rightarrow$ thiol-keto tautomerization of TT, the strongest IR active $\gamma_{\mathrm{e}}(\mathrm{CH})$ mode of a-SH ( $v_{32}$, see Figure S6c) is observed at $770.2 \mathrm{~cm}^{-1}$, i.e. $35-37 \mathrm{~cm}^{-1}$ higher in frequency than the similar mode of $\mathbf{s - O H}$.

Another important structural change in the TT molecule related with the tautomerization, is the change of the CS bond length. In the optimized geometry of s-OH, the CS bond is $169.4 \mathrm{pm}$, while for a-SH it elongates to $176.4 \mathrm{pm}$. This geometric change should be reflected in the value of the $v(\mathrm{CS})$ stretching frequency. A characteristic strong IR band due to the $v(\mathrm{C}=\mathrm{S})$ mode of $\mathbf{s}-\mathbf{O H}\left(v_{18}\right)$ appears at $1114 \mathrm{~cm}^{-1}$ coupled with vibrations of the ring. In both isomers of TT, also the $v_{23}$ mode has some contributions of $v(\mathrm{CS})$. For the a-SH band observed at $563.5 \mathrm{~cm}^{-1}$ (Figure S6c), the $v(\mathrm{C}-\mathrm{S})$ stretching mode has a dominant contribution, while the $\mathbf{s - O H}$ band observed at $599.8 \mathrm{~cm}^{-1}$ (Figure S6b) has a minor contribution of $v(\mathrm{C}=\mathrm{S})$, corresponding essentially to a ring deformation mode.

The computed spectrum suggests also that the $v_{6}$ mode, due to the $v(\mathrm{OH})$ stretching in the s-OH form (Figure S6a), might be used for the spectral identification of this form. However, the syn-OH group is involved in a very strong intramolecular H-bond. As it is typical of such cases, ${ }^{\mathrm{S} 2, \mathrm{~S} 3}$ the corresponding experimental absorption is very difficult to observe: this mode gives rise to a very broad absorption extended over several hundreds of wavenumbers, in the case of TT, approximately from 2900 to $2300 \mathrm{~cm}^{-1}$ (Figure S7a). This very broad feature disappears upon s-OH $\rightarrow$ a-SH tautomerization. However, its peak intensity is two orders of magnitude lower than the bands in the fingerprint range (see Figure S6b and Figure S7a) and this mode cannot be used for reliable spectral differentiation between s-OH and a-SH. As it is clear from Figure S7, the $v(\mathrm{CH})$ stretching modes $\left(v_{1}-v_{5}\right)$ in both isomers are also very weak, appearing at nearly the same frequencies (3200 to 2900 $\mathrm{cm}^{-1}$ ), and have similar band shapes; like the band due to $v(\mathrm{OH})$, they cannot be used to easily distinguish between the thione-enol and thiol-keto tautomers of TT. 


\section{Figures}
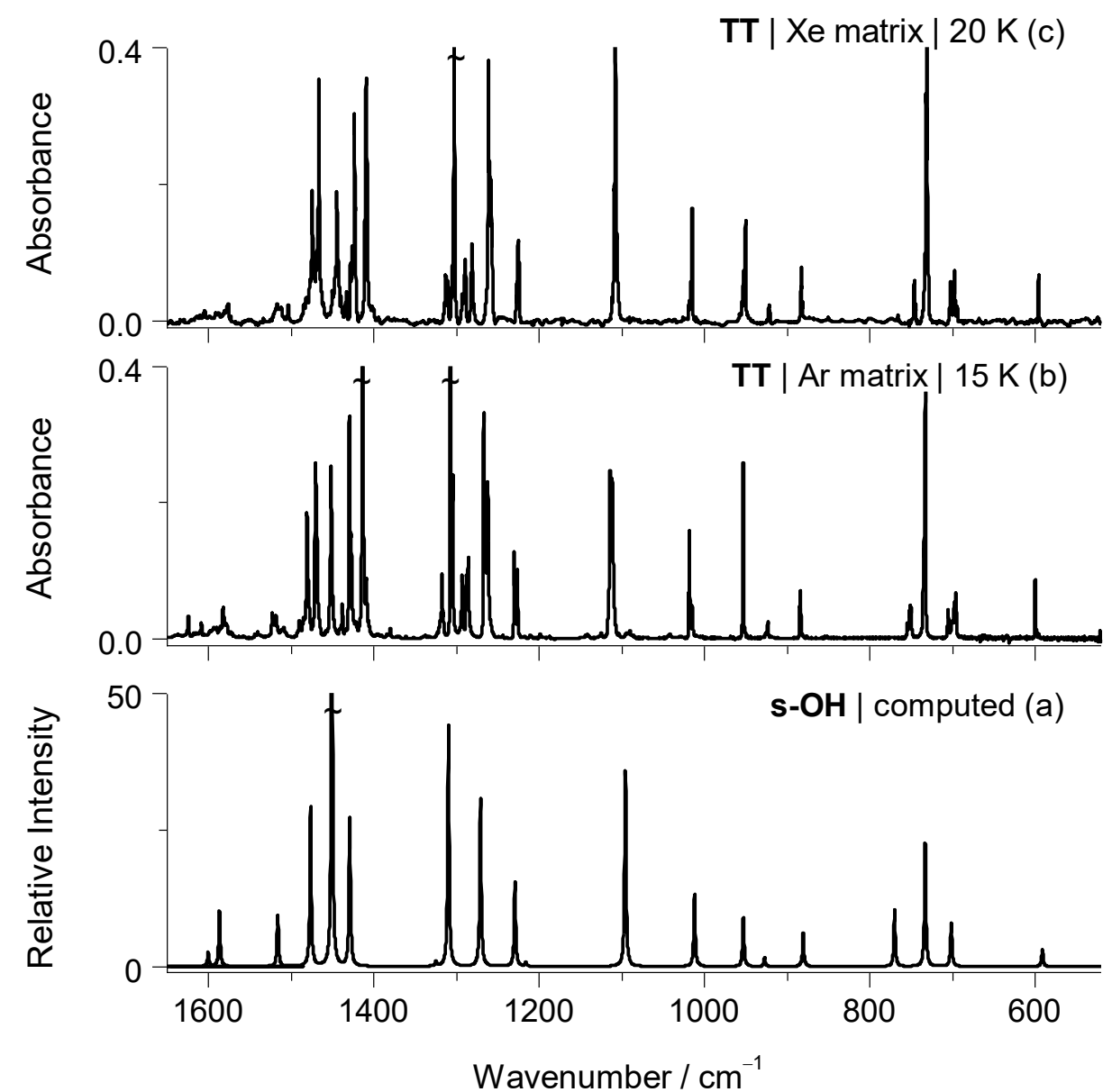

Figure S1. (a) B3LYP/6-311+G(2d,p) computed IR spectrum of the s-OH form of thiotropolone (TT). Experimental mid-IR spectra of TT: (b) monomers in argon matrix deposited at $15 \mathrm{~K}$; (c) monomers in xenon matrix deposited at $20 \mathrm{~K}$. 


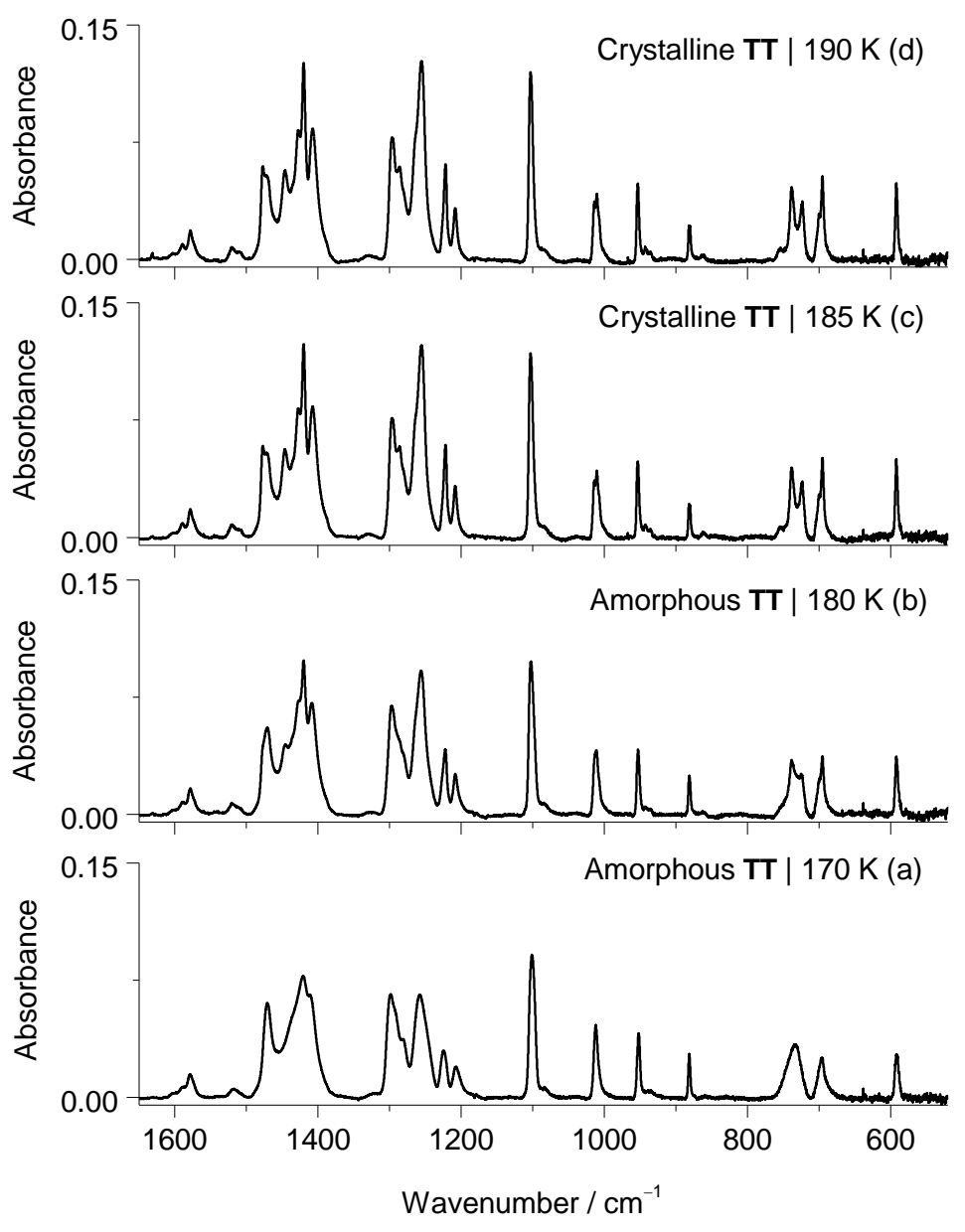

Figure S2. Experimental IR spectra of neat solid thiotropolone (TT) deposited at $15 \mathrm{~K}$ and annealed up to: (a) $170 \mathrm{~K}$; (b) $180 \mathrm{~K}$; (c) $185 \mathrm{~K}$; (d) $190 \mathrm{~K}$. The spectra were selected to display the temperature range where the thermally induced crystallization of TT occurred. 

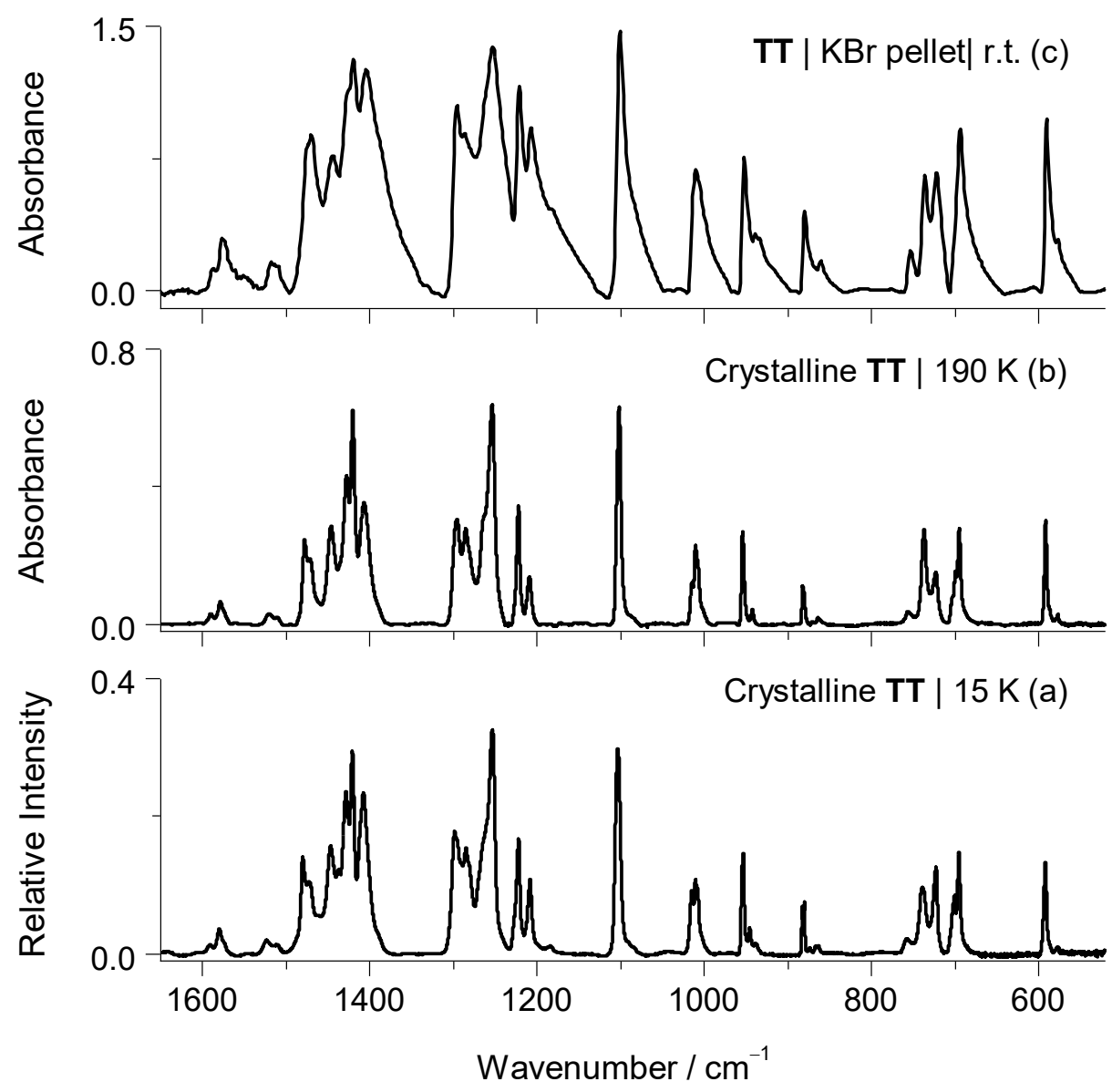

Figure S3. Experimental IR spectra of the neat solid of thiotropolone (TT) acquired from: (a) crystalline solid obtained after the sample has been deposited at $15 \mathrm{~K}$, annealed up to $190 \mathrm{~K}$, and cooled again to $15 \mathrm{~K}$; (b) crystalline solid of TT after the sample has been deposited at $190 \mathrm{~K}$; (c) solid sample of $\mathbf{T T}$ in a $\mathrm{KBr}$ pellet at room temperature (r.t.). 


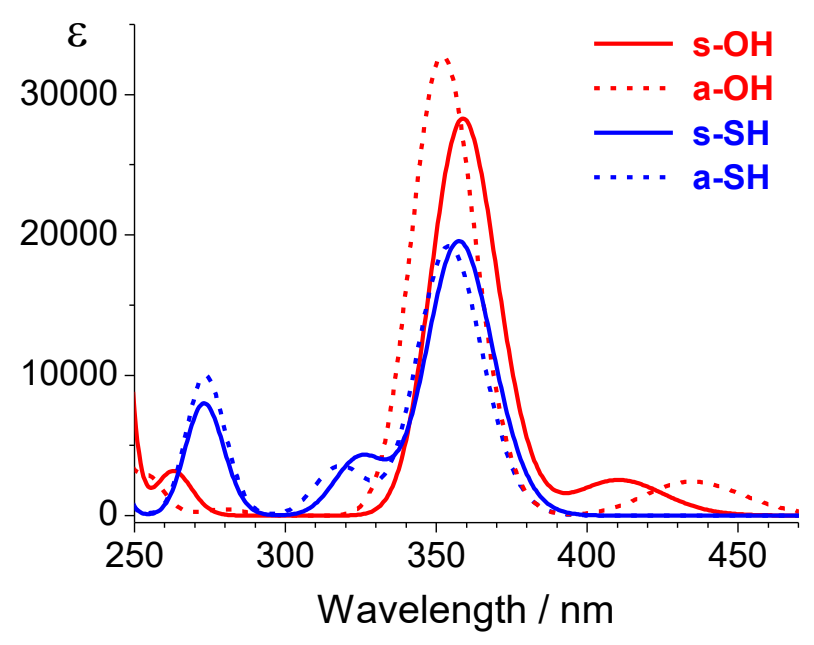

Figure S4. Simulated UV-Vis absorption spectra of both tautomers of thiotropolone, thione-enol (red) and thiol-keto (blue), based on the TD-DFT computations at the B3LYP/6-311+G(2d,p) level (see also Table S1). Solid lines correspond to the two syn forms (s-OH and s-SH) of each tautomeric stututure and dashed lines correspond to the two anti forms (a-OH and a-SH). 

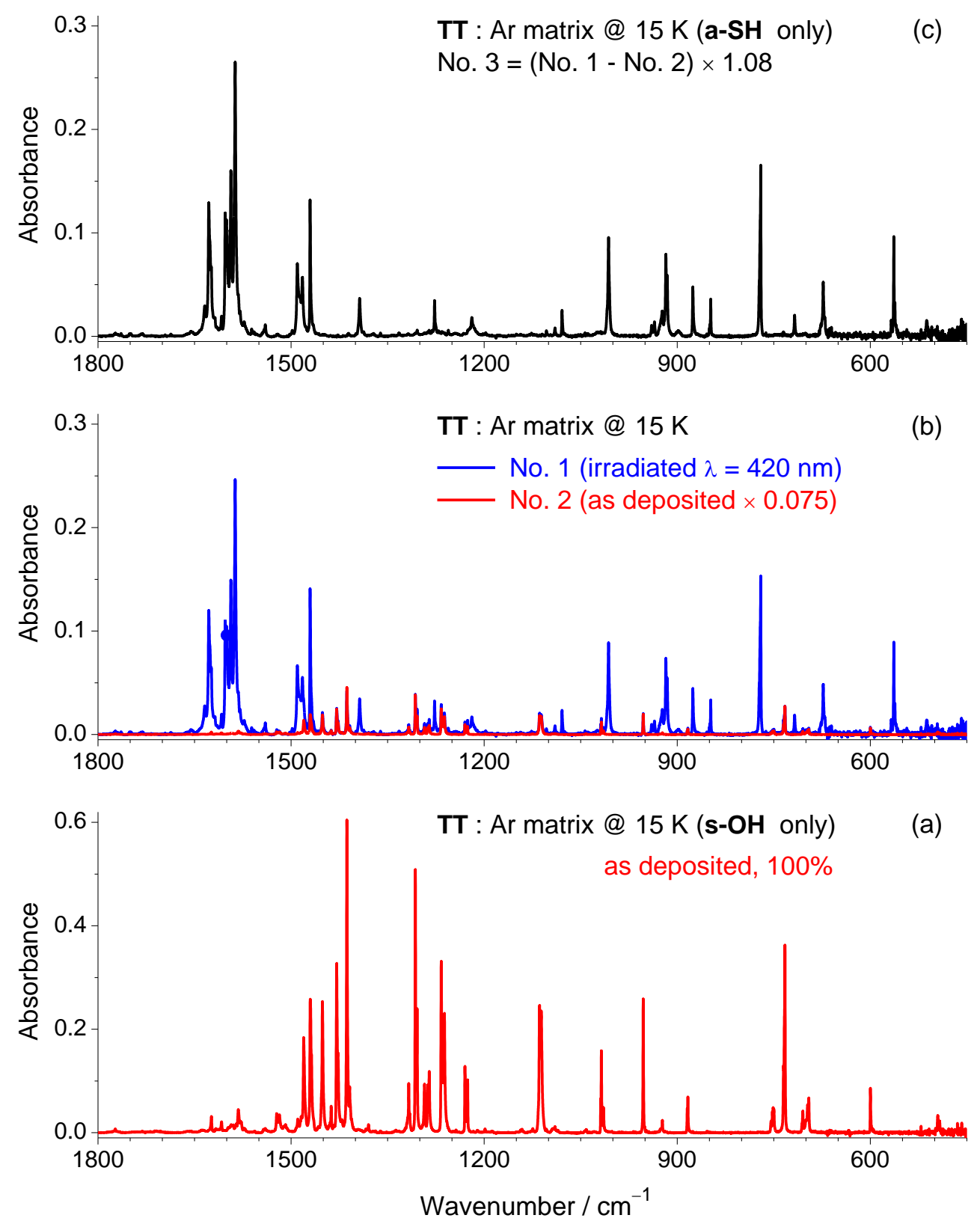

Figure S5. Experimental IR spectra of thiotropolone (TT) monomers isolated in an argon matrix at $15 \mathrm{~K}$ : (a) as deposited (s-OH form only), the same as in Figure 1b; (b) spectrum No.1 (blue) - after irradiation with visible light ( $\lambda=420 \mathrm{~nm}, 20 \mathrm{~mW}, 2 \mathrm{~min}$ ); No.2 (red) - the same as above, in frame S5a, but with intensity scaled by 0.075 , to match the bands of nonreacted s-OH form remaining in the sample after the irradiation; (c) spectrum No. 3 (black) where the contribution of non-reacted $\mathbf{s - O H}$ was nullified, and the resulting spectrum scaled up, to obtain the amount of photoproduct a-SH equimolar to the amount of reactant $\mathbf{s - O H}$ before the irradiation. 

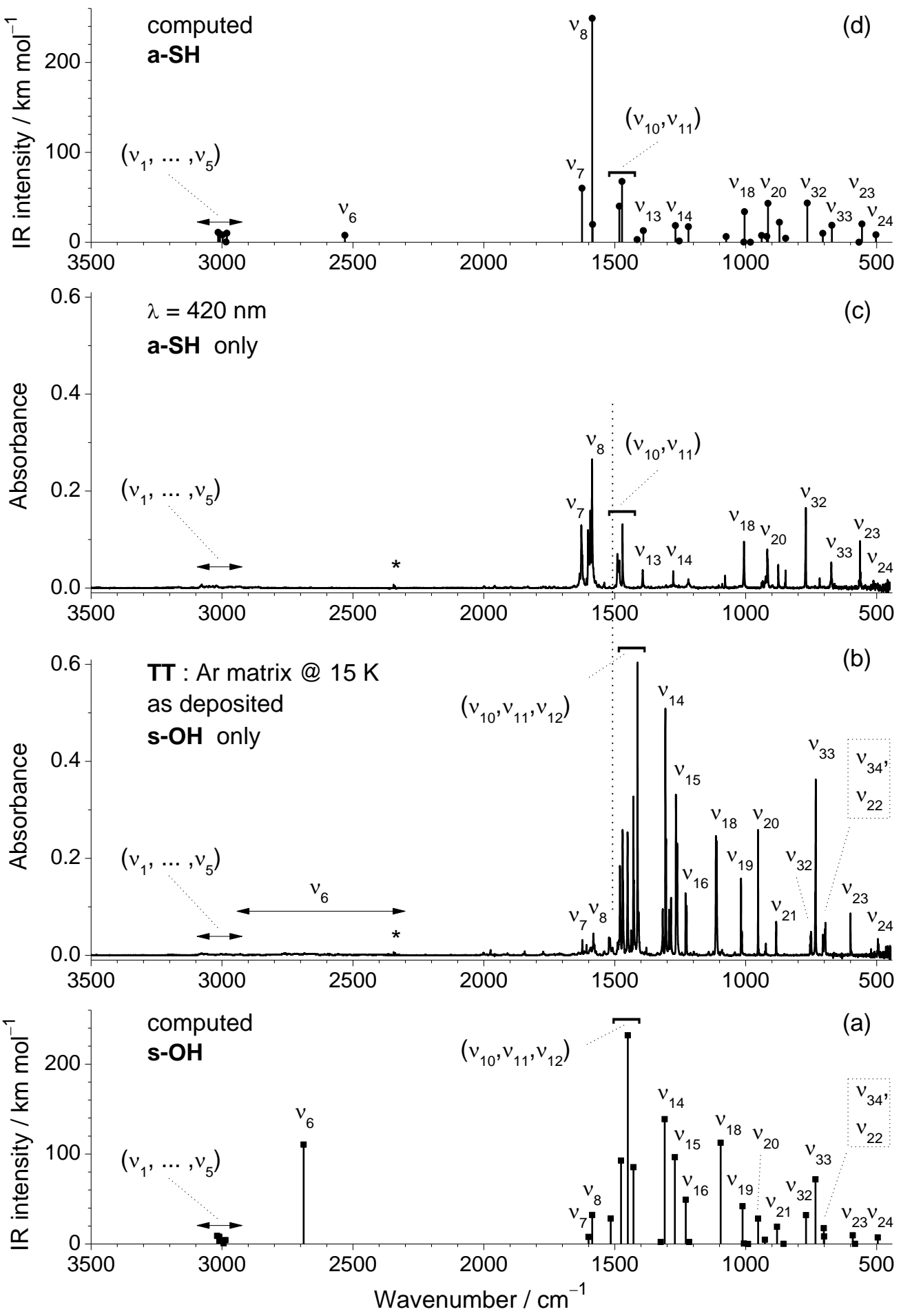

Figure S6. IR spectra of thiotropolone (TT) monomers computed at the B3LYP/6-311+G(2d,p) level of theory: (a) s-OH, and (d) a-SH; Experimental IR spectra of monomeric TT isolated in an argon matrix: (b) s-OH form only, and (c) a-SH form only (extracted as explained in Figure S5). Note that ordinate scales within pairs of frames (a,d) and (b,c) are intentionally equal. See Tables S2-S3 and S5$\mathrm{S} 6$ for the mode numbering and vibrational assignment. Asterisks in frames $(b, c)$ designate the band due to traces of atmospheric $\mathrm{CO}_{2}$ (the same band as in Figure $\mathrm{S} 7$ where this range is expanded). 

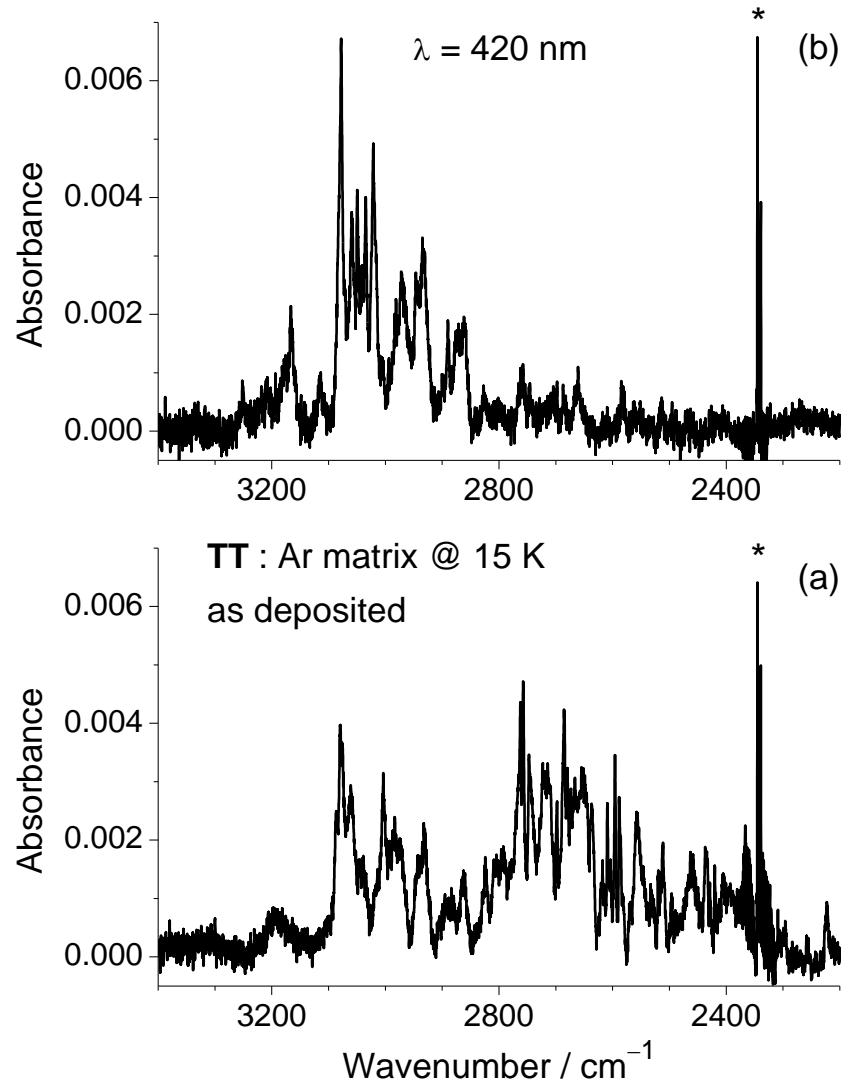

Figure S7. Experimental IR spectra of thiotropolone (TT) monomers isolated in an argon matrix at $15 \mathrm{~K}$ : (a) as deposited (s-OH form only), the same as in Figure S6b; (b) after irradiation with visible light $(\lambda=420 \mathrm{~nm}, 20 \mathrm{~mW}, 2 \mathrm{~min})$. The band marked with asterisk is due to the traces of atmospheric $\mathrm{CO}_{2}$ (same as in Figures S6b and S6c). 


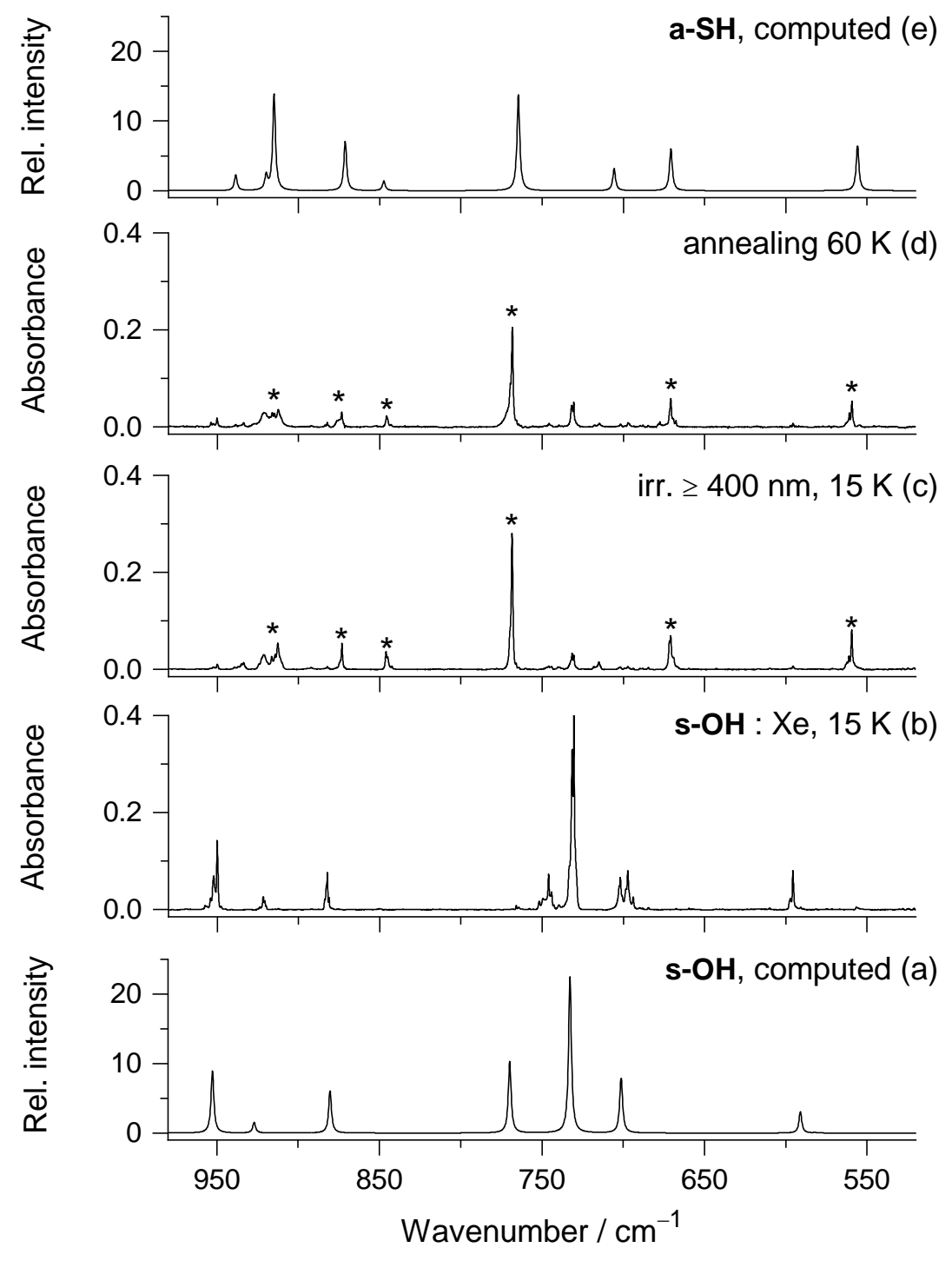

Figure S8. B3LYP/6-311+G(2d,p) computed IR spectra of (a) s-OH and (e) a-SH tautomers. Selected region of experimental mid-IR spectra of (b) thiotropolone s-OH freshly deposited in a Xe matrix at $15 \mathrm{~K}$; (c) after subsequent irradiation with $\lambda \geq 400 \mathrm{~nm}$; and (d) after subsequent annealing to $60 \mathrm{~K}$. Asterisks indicate bands of thiotropolone a-SH. 


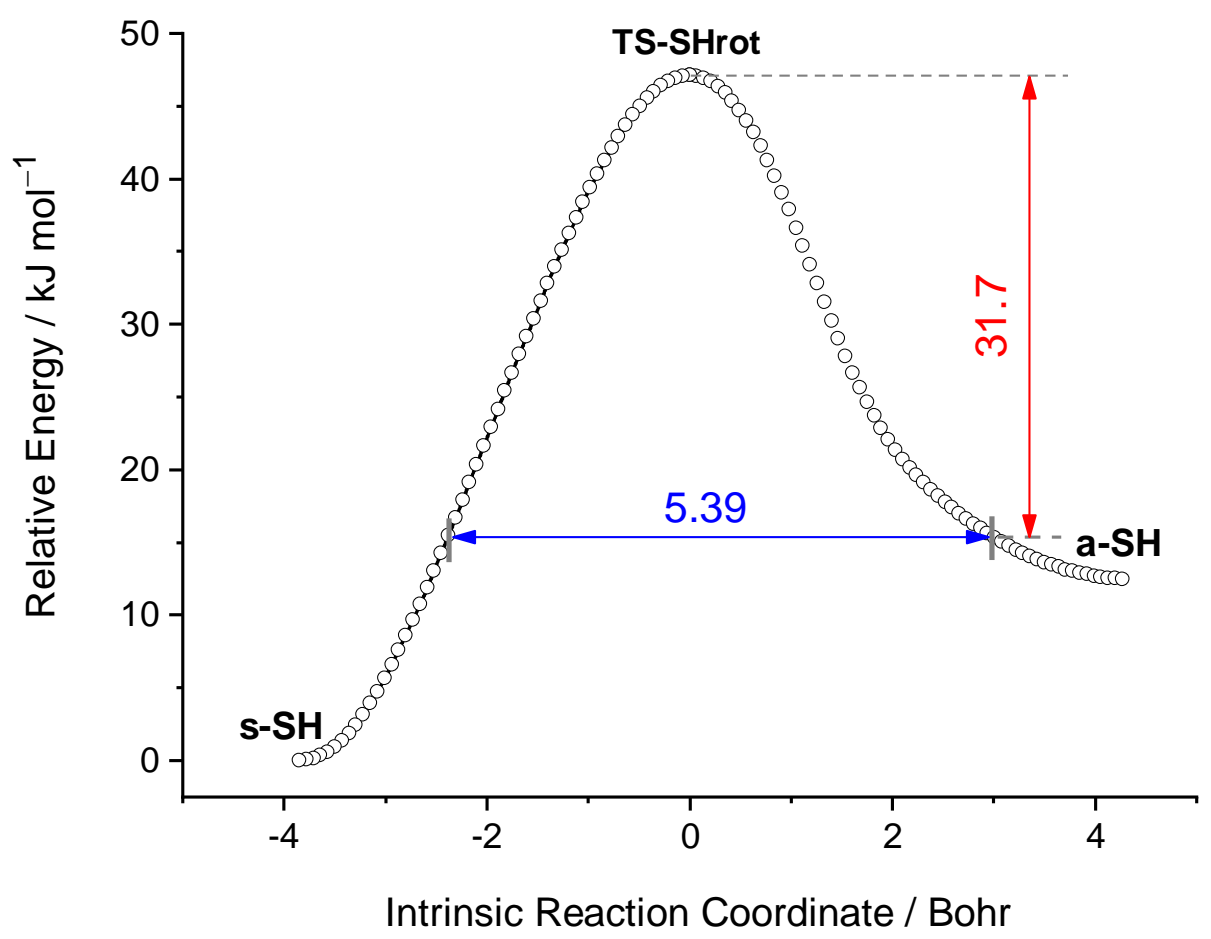

Figure S9. Relative electronic energy as a function of intrinsic reaction coordinate (IRC) for SH-rotamerization of a-SH to s-SH computed at the B3LYP/6-311+G(2d,p) level in non-mass-weighted (Cartesian) coordinates. The vertical arrow designates the calculated ZPE-corrected energy of the reactant a-SH relative to the transition state TS-SHrot. The horizontal arrow designates the barrier width considering the ZPE-corrected energy values of the stationary points superimposed with the pure electronic IRC energy profile. 


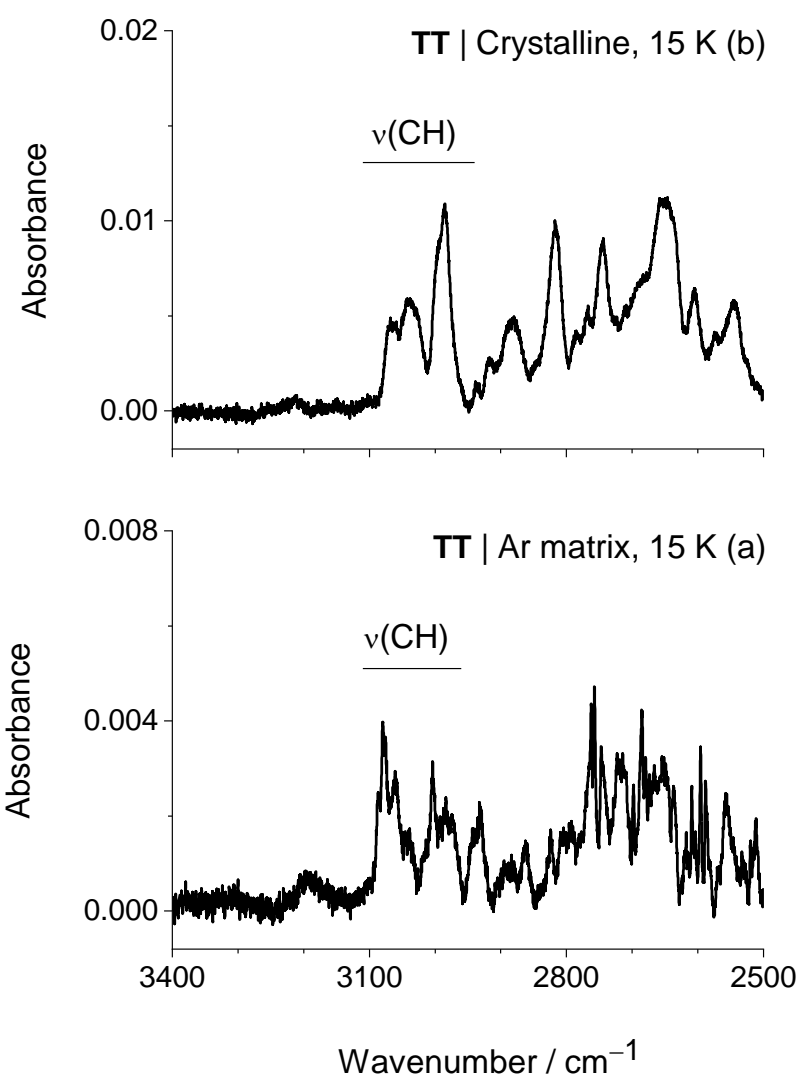

Figure S10. Selected region of the experimental IR spectra of TT: (a) monomeric compound deposited in an argon matrix at $15 \mathrm{~K}$; (b) neat compound at $15 \mathrm{~K}$ (crystalline solid). As indicated in the graphical part of the figure by horizontal lines, the IR bands due to $v(\mathrm{CH})$ stretching modes should appear in the region from 3100 to $2950 \mathrm{~cm}^{-1}$. The absorptions in the $2950-2500 \mathrm{~cm}^{-1}$ range should be due to the $v(\mathrm{OH})$ stretching mode. 


\section{Tables}

Table S1. Wavelengths (WL / nm) and oscillator strengths $(f)$ of the low-energy electronic excited singlet states computed at the B3LYP/6-311+G(2d,p) level using the time-dependent density functional theory (TD-DFT) for relevant species within the scope of this work.

\begin{tabular}{ccc}
\hline \multicolumn{3}{c}{ a-OH } \\
\hline WL & $f$ & Sym. \\
624.94 & 0.0000 & $\mathrm{~A}^{\prime \prime}$ \\
532.57 & 0.0000 & $\mathrm{~A}^{\prime}$ \\
434.95 & 0.0223 & $\mathrm{~A}^{\prime}$ \\
352.03 & 0.3014 & $\mathrm{~A}^{\prime}$ \\
279.69 & 0.0041 & $\mathrm{~A}^{\prime}$ \\
267.15 & 0.0004 & $\mathrm{~A}^{\prime \prime}$ \\
254.48 & 0.0224 & $\mathrm{~A}^{\prime}$ \\
250.99 & 0.0044 & $\mathrm{~A}^{\prime}$ \\
240.87 & 0.0003 & $\mathrm{~A}^{\prime \prime}$ \\
236.32 & 0.3960 & $\mathrm{~A}^{\prime}$ \\
233.63 & 0.0282 & $\mathrm{~A}^{\prime}$ \\
222.63 & 0.0006 & $\mathrm{~A}^{\prime \prime}$ \\
& & \\
\hline
\end{tabular}

\begin{tabular}{lcc}
\hline \multicolumn{3}{c}{ a-SH } \\
\hline WL & $f$ & Sym. \\
354.18 & 0.1766 & $\mathrm{~A}^{\prime}$ \\
345.80 & 0.0000 & $\mathrm{~A}^{\prime \prime}$ \\
323.61 & 0.0000 & $\mathrm{~A}^{\prime \prime}$ \\
318.27 & 0.0325 & $\mathrm{~A}^{\prime}$ \\
273.44 & 0.0918 & $\mathrm{~A}^{\prime}$ \\
249.55 & 0.0012 & $\mathrm{~A}^{\prime \prime}$ \\
235.28 & 0.3121 & $\mathrm{~A}^{\prime}$ \\
222.55 & 0.0000 & $\mathrm{~A}^{\prime \prime}$ \\
216.23 & 0.0114 & $\mathrm{~A}^{\prime \prime}$ \\
209.14 & 0.0304 & $\mathrm{~A}^{\prime}$ \\
203.89 & 0.0024 & $\mathrm{~A}^{\prime \prime}$ \\
202.72 & 0.0000 & $\mathrm{~A}^{\prime \prime}$ \\
& & \\
\hline
\end{tabular}

\begin{tabular}{ccc}
\hline \multicolumn{2}{c}{ s-OH } & \\
\hline WL & $f$ & Sym. \\
458.94 & 0.0000 & $\mathrm{~A}^{\prime \prime}$ \\
410.56 & 0.0233 & $\mathrm{~A}^{\prime}$ \\
407.71 & 0.0000 & $\mathrm{~A}^{\prime \prime}$ \\
358.89 & 0.2602 & $\mathrm{~A}^{\prime}$ \\
262.91 & 0.0292 & $\mathrm{~A}^{\prime}$ \\
243.35 & 0.0019 & $\mathrm{~A}^{\prime \prime}$ \\
240.09 & 0.4340 & $\mathrm{~A}^{\prime}$ \\
224.80 & 0.0078 & $\mathrm{~A}^{\prime}$ \\
217.39 & 0.0007 & $\mathrm{~A}^{\prime \prime}$ \\
213.35 & 0.0101 & $\mathrm{~A}^{\prime \prime}$ \\
207.93 & 0.0080 & $\mathrm{~A}^{\prime \prime}$ \\
207.18 & 0.0097 & $\mathrm{~A}^{\prime}$ \\
& & \\
\hline
\end{tabular}

\begin{tabular}{lcc}
\hline \multicolumn{3}{c}{ s-SH } \\
\hline WL & $f$ & Sym. \\
357.56 & 0.1797 & $\mathrm{~A}^{\prime}$ \\
328.18 & 0.0000 & $\mathrm{~A}^{\prime \prime}$ \\
325.33 & 0.0389 & $\mathrm{~A}^{\prime}$ \\
310.64 & 0.0000 & $\mathrm{~A}^{\prime \prime}$ \\
273.09 & 0.0736 & $\mathrm{~A}^{\prime}$ \\
243.10 & 0.0004 & $\mathrm{~A}^{\prime \prime}$ \\
235.78 & 0.3358 & $\mathrm{~A}^{\prime}$ \\
221.28 & 0.0028 & $\mathrm{~A}^{\prime \prime}$ \\
211.05 & 0.0194 & $\mathrm{~A}^{\prime \prime}$ \\
209.96 & 0.0002 & $\mathrm{~A}^{\prime \prime}$ \\
205.49 & 0.0000 & $\mathrm{~A}^{\prime \prime}$ \\
199.39 & 0.2093 & $\mathrm{~A}^{\prime}$ \\
& & \\
\hline
\end{tabular}


Table S2. Vibrational modes of the s-OH form of thiotropolone computed at the B3LYP/6$311+\mathrm{G}(2 \mathrm{~d}, \mathrm{p})$ level of theory in harmonic approximation, and vibrational assignment.

Mode Weight distribution (\%) of most prominent internal coordinates ${ }^{[a, b]} \begin{gathered}\text { Total } \\ (\%)\end{gathered} \quad \begin{gathered}\text { Total } \\ (\%)\end{gathered} \begin{gathered}\text { Approximate } \\ \text { Description }\end{gathered}$

\begin{tabular}{|c|c|c|c|c|}
\hline & A' symmetry & Bond & Angle & \\
\hline $\mathrm{S}_{1}$ & $\begin{array}{l}\mathrm{rC} 3 \mathrm{H} 11(12.6)+\mathrm{rC} 4 \mathrm{H} 12(19.7)+\mathrm{rC} 5 \mathrm{H} 13(\mathbf{3 2 . 1})+\mathrm{rC} 6 \mathrm{H} 14(15.0)+\mathrm{rC} 7 \mathrm{H} 15 \\
(12.4)\end{array}$ & 97.7 & 2.3 & $\mathrm{~V}_{\mathrm{a}}(\mathrm{CH})$ \\
\hline $\mathrm{S}_{2}$ & $\mathrm{rC} 3 \mathrm{H} 11(21.6)+\mathrm{rC} 4 \mathrm{H} 12(15.6)-\mathrm{rC} 6 \mathrm{H} 14(16.2)-\mathrm{rC} 7 \mathrm{H} 15$ (36.6) & 97.5 & 2.5 & $v_{\mathrm{b}}(\mathrm{CH})$ \\
\hline $\mathrm{S}_{3}$ & $\mathrm{rC} 3 \mathrm{H} 11(\mathbf{3 8 . 8})+\mathrm{rC} 4 \mathrm{H} 12(6.8)-\mathrm{rC} 5 \mathrm{H} 13(29.0)+\mathrm{rC} 7 \mathrm{H} 15$ (24.6) & 100.0 & 0.0 & $v_{\mathrm{c}}(\mathrm{CH})$ \\
\hline $\mathrm{S}_{4}$ & $\mathrm{rC} 3 \mathrm{H} 11(20.8)-\mathrm{rC} 4 \mathrm{H} 12(\mathbf{3 5 . 8})+\mathrm{rC} 5 \mathrm{H} 13(9.5)+\mathrm{rC} 6 \mathrm{H} 14(17.1)-\mathrm{rC} 7 \mathrm{H} 15(9.9)$ & 96.7 & 3.3 & $v_{\mathrm{d}}(\mathrm{CH})$ \\
\hline $\mathrm{S}_{5}$ & rC3H11 (6.6) - rC4H12 (15.3) + rC5H13 (17.8) - rC6H14 (40.6) + rC7H15 (14.6) & 98.3 & 1.7 & $v_{\mathrm{e}}(\mathrm{CH})$ \\
\hline $\mathrm{S}_{6}$ & $\mathrm{rO} 10 \mathrm{H} 9(\mathbf{6 4 . 9})+\mathrm{rC} 2 \mathrm{O} 10(5.7)+\beta \mathrm{C} 2 \mathrm{O} 10 \mathrm{H} 9(9.3)$ & 82.6 & 17.4 & $v(\mathrm{OH})$ \\
\hline $\mathrm{S}_{7}$ & $\begin{array}{l}\mathrm{rC} 2 \mathrm{C} 3(9.0)+\mathrm{rC} 3 \mathrm{C} 4(5.8)-\mathrm{rC} 4 \mathrm{C} 5(\mathbf{2 1 . 5})+\mathrm{rC} 6 \mathrm{C} 7(13.2)-\mathrm{rC} 7 \mathrm{C} 1(5.1) \\
-\beta \mathrm{C} 3 \mathrm{C} 4 \mathrm{H} 12(5.8)-\beta \mathrm{C} 6 \mathrm{C} 5 \mathrm{H} 13(5.5)\end{array}$ & 68.1 & 31.9 & $v_{a}(\mathrm{CC})$ \\
\hline $\mathrm{S}_{8}$ & $\mathrm{rC} 2 \mathrm{C} 3(\mathbf{1 7 . 5})-\mathrm{rC} 3 \mathrm{C} 4(14.1)+\mathrm{rC} 5 \mathrm{C} 6(13.6)-\operatorname{rC} 6 \mathrm{C} 7(\mathbf{1 6 . 2})-\beta \mathrm{C} 2 \mathrm{C} 3 \mathrm{H} 11(5.2)$ & 73.1 & 26.9 & $v_{\mathrm{b}}(\mathrm{CC})$ \\
\hline $\mathrm{S}_{9}$ & $\begin{array}{l}\mathrm{rC} 7 \mathrm{C} 1(\mathbf{1 1 . 9})-\mathrm{rC} 1 \mathrm{C} 2(8.3)+\mathrm{rC} 2 \mathrm{C} 3(6.8)+\mathrm{rC} 3 \mathrm{C} 4(7.0)-\mathrm{rC} 5 \mathrm{C} 6(5.5) \\
-\mathrm{rC} 6 \mathrm{C} 7(7.3)-\beta \mathrm{C} 2 \mathrm{O} 10 \mathrm{H} 9(\mathbf{1 0 . 3})-\beta \mathrm{C} 4 \mathrm{C} 5 \mathrm{H} 13(7.0)\end{array}$ & 53.6 & 46.4 & $\begin{array}{l}\delta(\mathrm{COH}) \\
-v_{b}(\mathrm{CC})\end{array}$ \\
\hline $\mathrm{S}_{10}$ & $\begin{array}{l}-\mathrm{rC} 7 \mathrm{C} 1(5.3)-\mathrm{rC} 2 \mathrm{C} 3(9.5)+\mathrm{rC} 3 \mathrm{C} 4(\mathbf{1 1 . 1})-\mathrm{rC} 4 \mathrm{C} 5(8.5)+\mathrm{rC} 5 \mathrm{C} 6(\mathbf{1 2 . 2}) \\
-\operatorname{rC} 6 \mathrm{C} 7(8.3)+\beta \mathrm{C} 2 \mathrm{C} 3 \mathrm{H} 11(8.4)-\beta \mathrm{C} 5 \mathrm{C} 6 \mathrm{H} 14(6.0)-\beta \mathrm{C} 2 \mathrm{O} 10 \mathrm{H} 9(5.2)\end{array}$ & 59.9 & 40.1 & $v_{c}(\mathrm{CC})+\delta_{\mathrm{a}}(\mathrm{CH})$ \\
\hline $\mathrm{S}_{11}$ & $\begin{array}{l}\mathrm{rC} 1 \mathrm{C} 2(10.1)+\mathrm{rC} 2 \mathrm{C} 3(6.8)-\mathrm{rC} 4 \mathrm{C} 5(\mathbf{1 2 . 4})-\mathrm{rC} 6 \mathrm{C} 7(11.6)+\mathrm{rC} 2 \mathrm{O} 10(5.6)+ \\
\beta \mathrm{C} 2 \mathrm{O} 10 \mathrm{H} 9(\mathbf{1 0 . 9})-\beta \mathrm{C} 1 \mathrm{C} 7 \mathrm{H} 15(8.5)+\beta \mathrm{C} 5 \mathrm{C} 4 \mathrm{H} 12(8.2)+\beta \mathrm{C} 4 \mathrm{C} 3 \mathrm{H} 11(6.1)\end{array}$ & 57.4 & 42.6 & $\begin{array}{l}\delta \mathrm{b}(\mathrm{CH})+ \\
\delta(\mathrm{COH}) \\
+v_{\mathrm{c}}(\mathrm{CC})\end{array}$ \\
\hline $\mathrm{S}_{12}$ & $\begin{array}{l}\beta \mathrm{C} 5 \mathrm{C} 4 \mathrm{H} 12(5.0)-\beta \mathrm{C} 4 \mathrm{C} 5 \mathrm{H} 13(\mathbf{1 1 . 5})-\beta \mathrm{C} 5 \mathrm{C} 6 \mathrm{H} 14(\mathbf{1 1 . 4})-\beta \mathrm{C} 6 \mathrm{C} 7 \mathrm{H} 15(9.8)+ \\
\beta \mathrm{C} 1 \mathrm{C} 2 \mathrm{O} 10(5.1)-\mathrm{rC} 7 \mathrm{C} 1(8.4)+\mathrm{rC} 2 \mathrm{C} 3(6.2)+\mathrm{rC} 4 \mathrm{C} 5(7.9)+\operatorname{rC} 6 \mathrm{C} 7(6.6)\end{array}$ & 35.6 & 64.4 & $\delta_{\mathrm{c}}(\mathrm{CH})$ \\
\hline $\mathrm{S}_{13}$ & $\begin{array}{l}-\mathrm{rC} 7 \mathrm{C} 1(\mathbf{1 2 . 9})+\mathrm{rC} 1 \mathrm{C} 2(\mathbf{1 2 . 9})+\mathrm{rC} 3 \mathrm{C} 4(5.9)-\mathrm{rC} 6 \mathrm{C} 7(8.9)-\mathrm{rC} 2 \mathrm{O} 10(\mathbf{1 1 . 0}) \\
-\beta \mathrm{C} 2 \mathrm{O} 10 \mathrm{H} 9(\mathbf{1 0 . 5})-\beta \mathrm{C} 1 \mathrm{C} 7 \mathrm{H} 15(6.7)\end{array}$ & 58.3 & 41.7 & $\begin{array}{l}v_{d}(\mathrm{CC})-v(\mathrm{CO}) \\
-\delta(\mathrm{COH})\end{array}$ \\
\hline $\mathrm{S}_{14}$ & $\begin{array}{l}\mathrm{rC} 2 \mathrm{O} 10(\mathbf{2 4 . 1})-\mathrm{rC} 7 \mathrm{C} 1(6.0)-\mathrm{rC} 2 \mathrm{C} 3(5.0)-\mathrm{rC} 3 \mathrm{C} 4(8.1)+\mathrm{rC} 6 \mathrm{C} 7(6.0) \\
+\beta \mathrm{C} 4 \mathrm{C} 3 \mathrm{H} 11(5.0)-\beta \mathrm{C} 3 \mathrm{C} 4 \mathrm{H} 12(6.4)+\beta \mathrm{C} 7 \mathrm{C} 6 \mathrm{H} 14(5.9)\end{array}$ & 60.6 & 39.4 & $v(\mathrm{CO})$ \\
\hline $\mathrm{S}_{15}$ & $\begin{array}{l}\beta \mathrm{C} 2 \mathrm{C} 3 \mathrm{H} 11(10.5)-\beta \mathrm{C} 1 \mathrm{C} 7 \mathrm{H} 15(6.6)+\beta \mathrm{C} 2 \mathrm{O} 10 \mathrm{H} 9(6.8)+\beta \mathrm{C} 1 \mathrm{C} 2 \mathrm{O} 10(6.3) \\
-\mathrm{rC} 7 \mathrm{C} 1(10.2)+\mathrm{rC} 2 \mathrm{C} 3(\mathbf{1 6 . 6})-\mathrm{rC} 3 \mathrm{C} 4(5.9)+\mathrm{rC} 6 \mathrm{C} 7(9.0)\end{array}$ & 48.3 & 51.7 & $\delta_{\mathrm{d}}(\mathrm{CH})$ \\
\hline $\mathrm{S}_{16}$ & $\begin{array}{l}-\mathrm{rC} 2 \mathrm{O} 10(\mathbf{1 6 . 3})+\mathrm{rC} 7 \mathrm{C} 1(10.9)-\mathrm{rC} 2 \mathrm{C} 3(5.1)+\mathrm{rC} 3 \mathrm{C} 4(5.0)+\mathrm{rC} 4 \mathrm{C} 5(9.8) \\
-\mathrm{rC} 5 \mathrm{C} 6(11.5)+\beta \mathrm{C} 4 \mathrm{C} 5 \mathrm{H} 13(6.9)+\beta \mathrm{C} 7 \mathrm{C} 6 \mathrm{H} 14(7.3)\end{array}$ & 67.4 & 32.6 & $v(\mathrm{CO})+v_{\mathrm{d}}(\mathrm{CC})$ \\
\hline $\mathrm{S}_{17}$ & $\begin{array}{l}\mathrm{rC} 6 \mathrm{C} 7(\mathbf{1 3 . 5})+\mathrm{rC} 7 \mathrm{C} 1(7.6)-\mathrm{rC} 1 \mathrm{C} 2(\mathbf{1 2 . 2})+\mathrm{rC} 3 \mathrm{C} 4(10.4)-\mathrm{rC} 4 \mathrm{C} 5(11.0) \\
-\mathrm{rC} 5 \mathrm{C} 6(5.6)+\beta \mathrm{C} 4 \mathrm{C} 3 \mathrm{H} 11(5.3)-\beta \mathrm{C} 5 \mathrm{C} 4 \mathrm{H} 12(6.4)-\beta \mathrm{C} 5 \mathrm{C} 6 \mathrm{H} 14(8.6)\end{array}$ & 65.6 & 34.4 & $v_{e}(\mathrm{CC})+\delta_{\mathrm{e}}(\mathrm{CH})$ \\
\hline $\mathrm{S}_{18}$ & $\begin{array}{l}\mathrm{rC} 6 \mathrm{C} 7(\mathbf{1 2 . 8})+\mathrm{rC} 7 \mathrm{C} 1(7.9)+\mathrm{rC} 1 \mathrm{C} 2(5.3)-\mathrm{rC} 3 \mathrm{C} 4(\mathbf{1 2 . 1})-\mathrm{rC} 4 \mathrm{C} 5(10.4) \\
+\mathrm{rC} 5 \mathrm{C} 6(5.0)-\mathrm{rC} 1 \mathrm{~S} 8(\mathbf{1 2 . 1})-\beta \mathrm{C} 1 \mathrm{C} 2 \mathrm{O} 10(5.6)-\beta \mathrm{C} 4 \mathrm{C} 5 \mathrm{H} 13(5.6)\end{array}$ & 73.0 & 27.0 & $v_{f}(\mathrm{CC})-v(\mathrm{CS})$ \\
\hline $\mathrm{S}_{19}$ & $\begin{array}{l}\mathrm{rC} 1 \mathrm{C} 2(5.5)+\mathrm{rC} 2 \mathrm{C} 3(\mathbf{1 1 . 4})+\mathrm{rC} 3 \mathrm{C} 4(9.3)-\mathrm{rC} 5 \mathrm{C} 6(\mathbf{1 0 . 3})-\mathrm{rC} 6 \mathrm{C} 7(7.1) \\
+\mathrm{rC} 2 \mathrm{O} 10(6.0)-\mathrm{rC} 1 \mathrm{~S} 8(\mathbf{1 0 . 7})+\beta \mathrm{C} 1 \mathrm{C} 7 \mathrm{C} 6(7.1)-\beta \mathrm{C} 2 \mathrm{C} 3 \mathrm{C} 4\end{array}$ & 65.5 & 34.5 & $v_{b}(C C)-v(C S)$ \\
\hline $\mathrm{S}_{20}$ & $\begin{array}{l}-\mathrm{rC} 3 \mathrm{C} 4(9.2)-\mathrm{rC} 4 \mathrm{C} 5(\mathbf{1 6 . 1})-\mathrm{rC} 5 \mathrm{C} 6(\mathbf{1 6 . 9})-\mathrm{rC} 6 \mathrm{C} 7(10.0)+\mathrm{rC} 1 \mathrm{~S} 8(7.1) \\
-\beta \mathrm{C} 1 \mathrm{C} 2 \mathrm{C} 3(5.9)\end{array}$ & 69.0 & 31.0 & $v_{\mathrm{g}}(\mathrm{CC})$ \\
\hline $\mathrm{S}_{21}$ & $\begin{array}{l}-\beta C 2 \mathrm{C} 3 \mathrm{C} 4(9.8)-\beta C 4 \mathrm{C} 5 \mathrm{C} 6(\mathbf{2 1 . 0})-\beta \mathrm{C} 1 \mathrm{C} 7 \mathrm{C} 6(7.6)+\mathrm{rC} 1 \mathrm{C} 2(14.7) \\
+\mathrm{rC} 4 \mathrm{C} 5(5.8)+\operatorname{rC} 5 \mathrm{C} 6(7.2)\end{array}$ & 43.1 & 56.9 & $\delta_{a}($ ring $)$ \\
\hline $\mathrm{S}_{22}$ & $\begin{array}{l}-\mathrm{rC} 2 \mathrm{O} 10(\mathbf{1 4 . 9})-\mathrm{rC} 7 \mathrm{C} 1(5.9)-\mathrm{rC} 1 \mathrm{C} 2(12.1)-\mathrm{rC} 2 \mathrm{C} 3(13.7)-\mathrm{rC} 3 \mathrm{C} 4(10.0) \\
+\beta \mathrm{C} 1 \mathrm{C} 7 \mathrm{C} 6(6.8)-\beta \mathrm{C} 7 \mathrm{C} 1 \mathrm{C} 2(5.1)\end{array}$ & 68.5 & 31.5 & $\begin{array}{l}\nu_{\mathrm{g}}(\mathrm{CC})+ \\
\delta_{\mathrm{b}}(\text { ring })\end{array}$ \\
\hline $\mathrm{S}_{23}$ & $\begin{array}{l}-\operatorname{rC} 1 S 8(\mathbf{2 0 . 3})-\mathrm{rC} 7 \mathrm{C} 1(14.1)-\mathrm{rC} 1 \mathrm{C} 2(16.0)-\mathrm{rC} 2 \mathrm{C} 3(8.0)-\mathrm{rC} 4 \mathrm{C} 5(5.3) \\
-\operatorname{rC} 6 \mathrm{C} 7(5.5)+\beta \mathrm{C} 2 \mathrm{C} 3 \mathrm{C} 4(7.0)+\beta \mathrm{C} 5 \mathrm{C} 6 \mathrm{C} 7(6.4)\end{array}$ & 77.3 & 22.7 & $v(\mathrm{CS})-v_{\mathrm{g}}(\mathrm{CC})$ \\
\hline $\mathrm{S}_{24}$ & $\begin{array}{l}-\beta \mathrm{C} 1 \mathrm{C} 2 \mathrm{O} 10(\mathbf{1 8 . 4})+\beta \mathrm{C} 3 \mathrm{C} 2 \mathrm{O} 10(10.1)+\beta \mathrm{C} 5 \mathrm{C} 6 \mathrm{C} 7(7.7)+\beta \mathrm{C} 1 \mathrm{C} 7 \mathrm{C} 6(5.9) \\
-\mathrm{rC} 7 \mathrm{C} 1(10.1)-\mathrm{rC} 1 \mathrm{C} 2(8.5)+\mathrm{rC} 2 \mathrm{C} 3(14.3)+\mathrm{rC} 3 \mathrm{C} 4(7.5)\end{array}$ & 46.8 & 53.2 & $\delta(\mathrm{CCO})$ \\
\hline $\mathrm{S}_{25}$ & $\begin{array}{l}\beta \mathrm{C} 1 \mathrm{C} 7 \mathrm{C} 6(\mathbf{1 0 . 5})-\beta \mathrm{C} 1 \mathrm{C} 2 \mathrm{C} 3(8.0)+\beta \mathrm{C} 2 \mathrm{C} 3 \mathrm{C} 4(5.5)+\beta \mathrm{C} 3 \mathrm{C} 4 \mathrm{C} 5(5.9) \\
-\beta \mathrm{C} 4 \mathrm{C} 5 \mathrm{C} 6(7.2)+\beta \mathrm{C} 7 \mathrm{C} 1 \mathrm{~S} 8(5.1)+\mathrm{rC} 7 \mathrm{C} 1(\mathbf{9 . 8})-\mathrm{rC} 1 \mathrm{C} 2(7.8)+\mathrm{rC} 3 \mathrm{C} 4(8.0) \\
+\operatorname{rC} 6 \mathrm{C} 7(8.0)+\mathrm{rC} 2 \mathrm{O} 10(8.1)\end{array}$ & 49.4 & 50.6 & $\delta_{c}($ ring $)$ \\
\hline $\mathrm{S}_{26}$ & $\begin{array}{l}\mathrm{rC} 1 \mathrm{~S} 8(\mathbf{1 5 . 0})+\mathrm{rC} 1 \mathrm{C} 2(10.1)+\mathrm{rC} 2 \mathrm{C} 3(6.3)-\mathrm{rC} 4 \mathrm{C} 5(4.9)+\beta \mathrm{C} 2 \mathrm{C} 1 \mathrm{~S} 8(\mathbf{1 3 . 3}) \\
-\beta \mathrm{C} 3 \mathrm{C} 2 \mathrm{O} 10(12.2)+\beta \mathrm{C} 2 \mathrm{C} 3 \mathrm{C} 4(11.2)+\beta \mathrm{C} 5 \mathrm{C} 6 \mathrm{C} 7(9.1)+\beta \mathrm{C} 1 \mathrm{C} 7 \mathrm{C} 6(7.1)\end{array}$ & 44.0 & 56.0 & $\delta_{\mathrm{d}}($ ring $)$ \\
\hline $\mathrm{S}_{27}$ & $\begin{array}{l}-\beta C 7 \mathrm{C} 1 \mathrm{~S} 8(\mathbf{2 0 . 4})+\beta \mathrm{C} 2 \mathrm{C} 1 \mathrm{~S} 8(6.9)+\beta \mathrm{C} 1 \mathrm{C} 7 \mathrm{C} 6(9.8)+\beta \mathrm{C} 1 \mathrm{C} 2 \mathrm{O} 10(9.1) \\
-\beta \mathrm{C} 1 \mathrm{C} 2 \mathrm{C} 3(\mathbf{7 . 0})+\mathrm{rC} 1 \mathrm{C} 2(11.5)+\mathrm{rC} 2 \mathrm{O} 10(7.1)-\mathrm{rC} 1 \mathrm{~S} 8(6.2)-\mathrm{rC} 6 \mathrm{C} 7(5.6)\end{array}$ & 38.8 & 61.2 & $\delta(\mathrm{CCS})$ \\
\hline
\end{tabular}




\begin{tabular}{|c|c|c|c|c|}
\hline & A" symmetry & Tors. & Out & \\
\hline $\mathrm{S}_{28}$ & $\begin{array}{l}\tau \mathrm{H} 14 \mathrm{C} 6 \mathrm{C} 7 \mathrm{H} 15(\mathbf{1 5 . 7})+\tau \mathrm{C} 2 \mathrm{C} 3 \mathrm{C} 4 \mathrm{C} 5(6.9)-\tau \mathrm{C} 3 \mathrm{C} 4 \mathrm{C} 5 \mathrm{C} 6(\mathbf{2 8 . 0})+\tau \mathrm{C} 4 \mathrm{C} 5 \mathrm{C} 6 \mathrm{C} 7 \\
(5.4)+\tau \mathrm{C} 6 \mathrm{C} 7 \mathrm{C} 1 \mathrm{C} 2(4.8)-\tau \mathrm{C} 7 \mathrm{C} 1 \mathrm{C} 2 \mathrm{C} 3(\mathbf{2 4 . 7})\end{array}$ & 94.3 & 5.7 & $\gamma_{\mathrm{a}}(\mathrm{CH})$ \\
\hline $\mathrm{S}_{29}$ & $\begin{array}{l}\tau \mathrm{C} 1 \mathrm{C} 2 \mathrm{C} 3 \mathrm{C} 4(27.7)-\tau \mathrm{C} 4 \mathrm{C} 5 \mathrm{C} 6 \mathrm{C} 7(6.1)+\tau \mathrm{C} 5 \mathrm{C} 6 \mathrm{C} 7 \mathrm{C} 1(19.2)-\tau \mathrm{C} 7 \mathrm{C} 1 \mathrm{C} 2 \mathrm{C} 3 \\
(17.8)-\tau \mathrm{C} 1 \mathrm{C} 2 \mathrm{C} 3 \mathrm{H} 11(8.0)-\tau \mathrm{H} 12 \mathrm{C} 4 \mathrm{C} 5 \mathrm{H} 13(9.8)-\tau \mathrm{H} 12 \mathrm{C} 4 \mathrm{C} 5 \mathrm{C} 6(5.7)\end{array}$ & 99.2 & 0.8 & $\gamma_{\mathrm{b}}(\mathrm{CH})$ \\
\hline $\mathrm{S}_{30}$ & $\begin{array}{l}-\tau \mathrm{C} 3 \mathrm{C} 4 \mathrm{C} 5 \mathrm{C} 6(8.7)+\tau \mathrm{C} 4 \mathrm{C} 5 \mathrm{C} 6 \mathrm{C} 7(8.7)-\tau \mathrm{C} 6 \mathrm{C} 7 \mathrm{C} 1 \mathrm{C} 2(\mathbf{1 5 . 5})-\tau \mathrm{H} 11 \mathrm{C} 3 \mathrm{C} 4 \mathrm{C} 5 \\
(12.4)+\tau \mathrm{C} 5 \mathrm{C} 6 \mathrm{C} 7 \mathrm{H} 15(12.1)+\tau \mathrm{S} 8 \mathrm{C} 1 \mathrm{C} 2 \mathrm{C} 3(11.4)-\tau \mathrm{C} 3 \mathrm{C} 4 \mathrm{C} 5 \mathrm{H} 13(6.0)\end{array}$ & 95.6 & 4.4 & $\gamma_{\mathrm{c}}(\mathrm{CH})$ \\
\hline $\mathrm{S}_{31}$ & $\begin{array}{l}-\tau \mathrm{C} 2 \mathrm{C} 3 \mathrm{C} 4 \mathrm{C} 5(11.8)+\tau \mathrm{C} 3 \mathrm{C} 4 \mathrm{C} 5 \mathrm{C} 6(11.8)+\tau \mathrm{C} 4 \mathrm{C} 5 \mathrm{C} 6 \mathrm{C} 7(\mathbf{1 5 . 4})-\tau \mathrm{C} 5 \mathrm{C} 6 \mathrm{C} 7 \mathrm{C} 1 \\
(6.9)-\tau \mathrm{C} 7 \mathrm{C} 1 \mathrm{C} 2 \mathrm{C} 3(8.4)-\tau \mathrm{C} 1 \mathrm{C} 7 \mathrm{C} 6 \mathrm{H} 14(11.1)-\tau \mathrm{C} 2 \mathrm{C} 3 \mathrm{C} 4 \mathrm{H} 12(8.6) \\
-\tau \mathrm{S} 8 \mathrm{C} 1 \mathrm{C} 7 \mathrm{C} 6(5.2)\end{array}$ & 91.1 & 8.9 & $\gamma_{\mathrm{d}}(\mathrm{CH})$ \\
\hline $\mathrm{S}_{32}$ & $\begin{array}{l}\gamma \mathrm{C} 1 \mathrm{C} 2 \mathrm{C} 3 \mathrm{O} 10(\mathbf{2 0 . 9})-\tau \mathrm{C} 1 \mathrm{C} 2 \mathrm{O} 10 \mathrm{H} 9(\mathbf{1 1 . 9})-\tau \mathrm{C} 3 \mathrm{C} 2 \mathrm{O} 10 \mathrm{H} 9(\mathbf{1 1 . 0})-\tau \mathrm{C} 7 \mathrm{C} 1 \mathrm{C} 2 \mathrm{C} 3 \\
(6.7)+\tau \mathrm{C} 1 \mathrm{C} 2 \mathrm{C} 3 \mathrm{C} 4(6.1)-\tau \mathrm{C} 2 \mathrm{C} 3 \mathrm{C} 4 \mathrm{H} 12(19.6)+\gamma \mathrm{C} 3 \mathrm{C} 4 \mathrm{C} 5 \mathrm{H} 12(15.3)\end{array}$ & 61.1 & 38.9 & $\tau \mathrm{OH}$ \\
\hline $\mathrm{S}_{33}$ & $\begin{array}{l}\gamma \mathrm{C} 4 \mathrm{C} 5 \mathrm{C} 6 \mathrm{H} 13(\mathbf{1 9 . 7})+\gamma \mathrm{C} 5 \mathrm{C} 6 \mathrm{C} 7 \mathrm{H} 14(6.7)+\gamma \mathrm{C} 3 \mathrm{C} 4 \mathrm{C} 5 \mathrm{H} 12(5.9)+\tau \mathrm{C} 1 \mathrm{C} 2 \mathrm{C} 3 \mathrm{C} 4 \\
(5.8)+\tau \mathrm{H} 13 \mathrm{C} 5 \mathrm{C} 6 \mathrm{C} 7(9.7)+\tau \mathrm{C} 2 \mathrm{C} 3 \mathrm{C} 4 \mathrm{C} 5(16.3)-\tau \mathrm{C} 6 \mathrm{C} 7 \mathrm{C} 1 \mathrm{C} 2(7.4) \\
-\tau \mathrm{S} 8 \mathrm{C} 1 \mathrm{C} 7 \mathrm{C} 6(5.0)+\tau \mathrm{O} 10 \mathrm{C} 2 \mathrm{C} 3 \mathrm{C} 4(7.4)\end{array}$ & 60.4 & 39.6 & $\gamma_{\mathrm{e}}(\mathrm{CH})$ \\
\hline $\mathrm{S}_{34}$ & $\begin{array}{l}\tau \mathrm{C} 5 \mathrm{C} 6 \mathrm{C} 7 \mathrm{C} 1(\mathbf{2 0 . 7})-\tau \mathrm{C} 6 \mathrm{C} 7 \mathrm{C} 1 \mathrm{C} 2(13.1)-\tau \mathrm{C} 1 \mathrm{C} 2 \mathrm{C} 3 \mathrm{C} 4(12.1)+\tau \mathrm{C} 2 \mathrm{C} 3 \mathrm{C} 4 \mathrm{C} 5 \\
(\mathbf{1 9 . 0})-\tau \mathrm{C} 4 \mathrm{C} 5 \mathrm{C} 6 \mathrm{C} 7(6.8)+\tau \mathrm{S} 8 \mathrm{C} 1 \mathrm{C} 7 \mathrm{C} 6(5.2)\end{array}$ & 92.4 & 7.6 & $\tau_{\mathrm{a}}($ ring $)$ \\
\hline $\mathrm{S}_{35}$ & $\begin{array}{l}\tau \mathrm{C} 5 \mathrm{C} 6 \mathrm{C} 7 \mathrm{C} 1(\mathbf{1 4 . 7})+\tau \mathrm{C} 1 \mathrm{C} 2 \mathrm{C} 3 \mathrm{C} 4(8.3)-\tau \mathrm{C} 2 \mathrm{C} 3 \mathrm{C} 4 \mathrm{C} 5(12.2)+\tau \mathrm{C} 3 \mathrm{C} 4 \mathrm{C} 5 \mathrm{C} 6 \\
(\mathbf{1 6 . 6})-\tau \mathrm{C} 4 \mathrm{C} 5 \mathrm{C} 6 \mathrm{C} 7(\mathbf{1 7 . 3})-\tau \mathrm{S} 8 \mathrm{C} 1 \mathrm{C} 7 \mathrm{C} 6(6.2)\end{array}$ & 91.1 & 8.9 & $\tau_{\mathrm{b}}($ ring $)$ \\
\hline $\mathrm{S}_{36}$ & $\begin{array}{l}\tau \mathrm{C} 7 \mathrm{C} 1 \mathrm{C} 2 \mathrm{C} 3(9.0)-\tau \mathrm{C} 1 \mathrm{C} 2 \mathrm{C} 3 \mathrm{C} 4(14.0)-\tau \mathrm{C} 2 \mathrm{C} 3 \mathrm{C} 4 \mathrm{H} 12(\mathbf{2 2 . 2})+\tau \mathrm{H} 12 \mathrm{C} 4 \mathrm{C} 5 \mathrm{C} 6 \\
(7.7)+\gamma \mathrm{C} 3 \mathrm{C} 4 \mathrm{C} 5 \mathrm{H} 12(\mathbf{2 4 . 7})+\gamma \mathrm{C} 1 \mathrm{C} 2 \mathrm{C} 3 \mathrm{O} 10(5.7)\end{array}$ & 64.1 & 35.9 & $\tau_{c}($ ring $)$ \\
\hline $\mathrm{S}_{37}$ & $\begin{array}{l}\gamma \mathrm{C} 5 \mathrm{C} 6 \mathrm{C} 7 \mathrm{H} 14(\mathbf{2 7 . 1})+\tau \mathrm{C} 1 \mathrm{C} 7 \mathrm{C} 6 \mathrm{H} 14(\mathbf{2 6 . 6})+\tau \mathrm{C} 6 \mathrm{C} 7 \mathrm{C} 1 \mathrm{C} 2(9.7)-\tau \mathrm{C} 7 \mathrm{C} 1 \mathrm{C} 2 \mathrm{C} 3 \\
(7.2)-\tau \mathrm{C} 4 \mathrm{C} 5 \mathrm{C} 6 \mathrm{H} 14(7.1)-\tau \mathrm{S} 8 \mathrm{C} 1 \mathrm{C} 2 \mathrm{C} 3(5.7)\end{array}$ & 66.5 & 33.5 & $\tau_{\mathrm{d}}($ ring $)$ \\
\hline & $\gamma \mathrm{C} 1 \mathrm{C} 2 \mathrm{C} 3 \mathrm{O} 10(\mathbf{1 9 . 3})-\gamma \mathrm{C} 2 \mathrm{C} 1 \mathrm{C} 7 \mathrm{~S} 8(\mathbf{1 1 . 8})-\tau \mathrm{C} 7 \mathrm{C} 1 \mathrm{C} 2 \mathrm{O} 10(\mathbf{1 5 . 8})$ & 66.7 & 33.3 & $\gamma(\mathrm{CO})-\gamma(\mathrm{CS})$ \\
\hline $\mathrm{S}_{38}$ & $\begin{array}{l}+\tau \mathrm{S} 8 \mathrm{C} 1 \mathrm{C} 2 \mathrm{C} 3(\mathbf{1 2 . 7})+\tau \mathrm{C} 5 \mathrm{C} 6 \mathrm{C} 7 \mathrm{C} 1(10.0)-\tau \mathrm{O} 10 \mathrm{C} 2 \mathrm{C} 3 \mathrm{H} 11(7.6)-\tau \mathrm{C} 3 \mathrm{C} 4 \mathrm{C} 5 \mathrm{C} 6 \\
(5.6)-\tau \mathrm{H} 11 \mathrm{C} 3 \mathrm{C} 4 \mathrm{C} 5(5.5)\end{array}$ & & & \\
\hline $\mathrm{S}_{39}$ & $\begin{array}{l}\tau \mathrm{S} 8 \mathrm{C} 1 \mathrm{C} 2 \mathrm{O} 10(\mathbf{2 5 . 2})+\gamma \mathrm{C} 2 \mathrm{C} 1 \mathrm{C} 7 \mathrm{~S} 8(\mathbf{1 8 . 1})-\tau \mathrm{C} 4 \mathrm{C} 5 \mathrm{C} 6 \mathrm{C} 7(17.6)+\tau \mathrm{C} 2 \mathrm{C} 3 \mathrm{C} 4 \mathrm{C} 5 \\
(8.8)-\tau \mathrm{C} 3 \mathrm{C} 4 \mathrm{C} 5 \mathrm{C} 6(7.3)\end{array}$ & 79.5 & 20.5 & $\gamma(\mathrm{CS})+\gamma(\mathrm{CO})$ \\
\hline
\end{tabular}

[a] See Scheme S1 for the atom numbering. Internal coordinates used in the analysis of vibrational modes are defined automatically, as described in ref. S4.

[b] All contributions of most prominent internal coordinates, with weights of 5\% or higher, are included. The top contributions are shown in bold. Abbreviations: $v=$ stretching, $\delta=$ in-plane bending, $\gamma=$ out-of-plane bending, $\tau=$ torsion; $r_{i, j}$ is the distance between atoms $A_{i}$ and $A_{j} ; \beta_{i, j, k}$ is the angle between vectors $A_{k} A_{i}$ and $A_{k} A_{j} ; \tau_{i, j, k, l}$ is the dihedral angle between the plane defined by $A_{i}, A_{j}, A_{k}$ and the plane defined by $A_{j}, A_{k}$ and $A_{l}$ atoms; $\gamma_{i, j, k, l}$ is the angle between the vector $A_{k} A_{i}$ and the plane defined by atoms $A_{j}, A_{k}, A_{l}$. The symbols $(+)$ and $(-)$ denote in-phase and in-opposite-phase couplings between coordinates of different types.

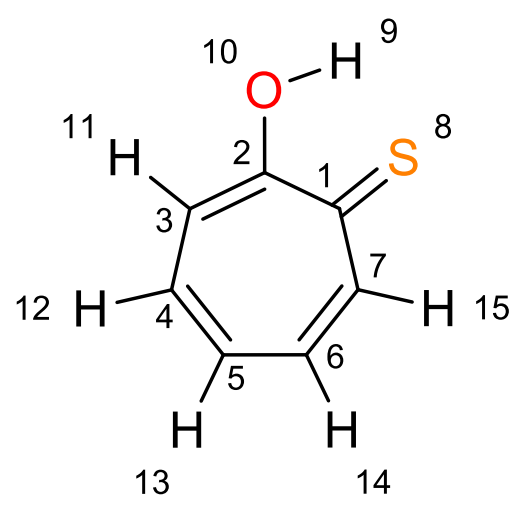

Scheme S1. Structure and the atom numbering of the s-OH form of thiotropolone. 
Table S3. Experimental IR spectra of s-OH form of TT isolated in an Ar matrix at $15 \mathrm{~K}$, and in neat TT crystals at $15 \mathrm{~K}$, compared with harmonic and anharmonic wavenumbers $\left(\tilde{v} / \mathrm{cm}^{-1}\right)$ and infrared intensities $\left(I / \mathrm{km} \mathrm{mol}^{-1}\right)$ computed at the B3LYP/6-311+G(2d,p) level of theory, and vibrational assignment.

\begin{tabular}{|c|c|c|c|c|c|c|c|c|c|}
\hline \multirow{2}{*}{$\begin{array}{l}\text { Mode } \\
\mathrm{N}^{\circ} .\end{array}$} & \multicolumn{2}{|c|}{ Ar matrix } & \multirow{2}{*}{$\frac{\frac{\text { Crystal }}{}^{[\mathrm{a}]}}{\tilde{v}_{e x p}}$} & \multicolumn{3}{|c|}{ Calc. $^{[\mathrm{b}]}$} & \multirow[b]{2}{*}{$I_{\text {anhar }}$} & \multirow[t]{2}{*}{ Sym. } & \multirow{2}{*}{$\begin{array}{l}\text { Approximate } \\
\text { Description }^{[\mathrm{c}]}\end{array}$} \\
\hline & $\tilde{v}_{e x p}$ & $I_{\text {exp }}$ & & $\tilde{v}_{\text {harm }}$ & $I_{\text {harm }}$ & $\tilde{v}_{\text {anhar }}$ & & & \\
\hline \multicolumn{10}{|c|}{$A^{\prime}$ symmetry } \\
\hline$v_{8}+v_{10}$ & & & & & & 3091.9 & 6.4 & $\mathrm{~A}^{\prime}$ & \\
\hline$v_{7}+v_{10}$ & & & & & & 3075.8 & 5.6 & $\mathrm{~A}^{\prime}$ & \\
\hline$v_{1}$ & & - & & 3018.4 & 8.9 & 3050.7 & 18.0 & $\mathrm{~A}^{\prime}$ & $v_{a}(\mathrm{CH})$ \\
\hline$v_{2}$ & & - & & 3010.7 & 8.1 & 2991.9 & 15.7 & $\mathrm{~A}^{\prime}$ & $v_{b}(\mathrm{CH})$ \\
\hline$v_{3}$ & & - & & 3007.4 & 3.8 & 3047.1 & 12.4 & $\mathrm{~A}^{\prime}$ & $v_{c}(\mathrm{CH})$ \\
\hline$v_{4}$ & & - & & 2994.0 & 0.7 & 3037.8 & 2.4 & $\mathrm{~A}^{\prime}$ & $v_{\mathrm{d}}(\mathrm{CH})$ \\
\hline$v_{5}$ & & - & & 2986.9 & 4.3 & 3001.8 & 38.6 & $\mathrm{~A}^{\prime}$ & $v_{\mathrm{e}}(\mathrm{CH})$ \\
\hline$v_{8}+v_{12}$ & & & & & & 2995.6 & 5.4 & $\mathrm{~A}^{\prime}$ & \\
\hline$v_{7}+v_{12}$ & & & & & & 2987.5 & 10.5 & $\mathrm{~A}^{\prime}$ & \\
\hline$v_{9}+v_{10}$ & & & & & & 2983.2 & 13.7 & $\mathrm{~A}^{\prime}$ & \\
\hline$v_{6}$ & & & & 2961.8 & 200.3 & 2688.1 & 110.5 & $\mathrm{~A}^{\prime}$ & $v(\mathrm{OH})$ \\
\hline$v_{31}+v_{33}$ & & & & & & 1613.8 & 5.4 & $\mathrm{~A}^{\prime}$ & \\
\hline$v_{19}+v_{23}$ & & & & & & 1613.5 & 11.9 & $\mathrm{~A}^{\prime}$ & \\
\hline$v_{18}+v_{24}$ & & & & & & 1603.8 & 11.2 & $\mathrm{~A}^{\prime}$ & \\
\hline$v_{7}$ & $\sim 1600 \mathrm{ov} .{ }^{[\mathrm{d}]}$ & & 1590 & 1602.0 & 7.9 & 1595.9 & 2.2 & $\mathrm{~A}^{\prime}$ & $v_{a}(C=C)$ \\
\hline$v_{21}+v_{22}$ & & & & & & 1588.2 & 8.6 & $\mathrm{~A}^{\prime}$ & \\
\hline$v_{8}$ & 1581.9 & 24.1 & 1579 & 1588.0 & 32.3 & 1582.5 & 0.4 & $\mathrm{~A}^{\prime}$ & $v_{b}(C=C)$ \\
\hline $2 \times v_{32}$ & & & & & & 1571.6 & 54.3 & $\mathrm{~A}^{\prime}$ & $2 \times \tau \mathrm{OH}$ \\
\hline$v_{32}+v_{34}$ & & & & & & 1551.7 & 51.6 & $\mathrm{~A}^{\prime}$ & \\
\hline$v_{32}+v_{33}$ & & & & & & 1528.4 & 19.0 & $\mathrm{~A}^{\prime}$ & \\
\hline$v_{19}+v_{24}$ & & & & & & 1520.0 & 7.4 & $\mathrm{~A}^{\prime}$ & \\
\hline$v_{9}$ & $\mathbf{1 5 2 2 . 4}, 1518.2$ & 20.5 & $\mathbf{1 5 2 3}, 1511$ & 1517.4 & 28.3 & 1504.5 & 26.1 & $\mathrm{~A}^{\prime}$ & $\delta(\mathrm{COH})-v_{\mathrm{b}}(\mathrm{C}=\mathrm{C})$ \\
\hline$v_{31}+v_{35}$ & & & & & & 1488.9 & 21.0 & $\mathrm{~A}^{\prime}$ & \\
\hline$v_{10}$ & 1480.3 & 56.9 & 1479 & 1477.5 & 92.7 & 1477.1 & 40.7 & $\mathrm{~A}^{\prime}$ & $v_{c}(\mathrm{C}=\mathrm{C})+\delta_{\mathrm{a}}(\mathrm{CH})$ \\
\hline$v_{21}+v_{23}$ & 1470.1 & 78.7 & 1471 & & & 1478.9 & 12.2 & $\mathrm{~A}^{\prime}$ & \\
\hline \multirow[t]{2}{*}{$v_{11}$} & 1451.3 & 64.5 & 1447 & 1451.8 & 232.1 & 1425.5 & 132.3 & $\mathrm{~A}^{\prime}$ & $\delta_{b}(\mathrm{CH})+\delta(\mathrm{COH})$ \\
\hline & 1437.7 & 11.7 & 1436 & & & & & & \\
\hline \multirow[t]{2}{*}{$v_{12}$} & 1429.1 & 93.3 & $1428, \mathbf{1 4 2 1}$ & 1430.3 & 85.3 & 1426.3 & 121.5 & $\mathrm{~A}^{\prime}$ & $\delta_{\mathrm{c}}(\mathrm{CH})$ \\
\hline & 1413.3 & 126.7 & 1408 & & & & & & \\
\hline$v_{34}+v_{35}$ & & & & & & 1388.8 & 9.8 & $\mathrm{~A}^{\prime}$ & \\
\hline$v_{30}+v_{36}$ & 1317.1 & 23.4 & & & & 1332.9 & 28.0 & $\mathrm{~A}^{\prime}$ & \\
\hline$v_{13}$ & & & & 1325.6 & 2.2 & 1307.0 & 4.8 & $\mathrm{~A}^{\prime}$ & $v_{d}(\mathrm{CC})-v(\mathrm{CO})-\delta(\mathrm{COH})$ \\
\hline$v_{14}$ & 1306.8, 1285.4 & 154.6 & $\mathbf{1 2 9 8}, 1285$ & 1310.7 & 138.7 & 1314.6 & 91.3 & $\mathrm{~A}^{\prime}$ & $v(\mathrm{C}-\mathrm{O})$ \\
\hline$v_{15}$ & 1266.7, 1261.8 & 113.3 & 1253 & 1271.6 & 96.5 & 1272.6 & 98.7 & $\mathrm{~A}^{\prime}$ & $\delta_{\mathrm{d}}(\mathrm{CH})$ \\
\hline$v_{20}+v_{27}$ & & & & & & 1266.5 & 6.8 & $\mathrm{~A}^{\prime}$ & \\
\hline$v_{16}$ & 1229.8, 1225.7 & 30.2 & 1222 & 1230.3 & 49.2 & 1234.2 & 42.3 & $\mathrm{~A}^{\prime}$ & $v(\mathrm{CO})+v_{\mathrm{d}}(\mathrm{CC})$ \\
\hline$v_{17}$ & & - & 1208 & 1217.1 & 2.2 & 1218.4 & 10.5 & $\mathrm{~A}^{\prime}$ & $v_{e}(\mathrm{CC})+\delta_{e}(\mathrm{CH})$ \\
\hline$v_{30}+v_{38}$ & & & & & & 1104.3 & 5.5 & $\mathrm{~A}^{\prime}$ & \\
\hline$v_{18}$ & 1113.9, 1111.3 & 105.9 & 1103 & 1097.0 & 112.6 & 1101.2 & 88.4 & $\mathrm{~A}^{\prime}$ & $v_{f}(C C)-v(C S)$ \\
\hline$v_{23}+v_{24}$ & & & & & & 1093.7 & 8.1 & $\mathrm{~A}^{\prime}$ & \\
\hline$v_{19}$ & 1017.9, 1014.7 & 27.5 & $1014, \mathbf{1 0 1 0}$ & 1013.0 & 42.0 & 1017.0 & 24.0 & $\mathrm{~A}^{\prime}$ & $v_{b}(\mathrm{CC})-v(\mathrm{CS})$ \\
\hline$v_{23}+v_{25}$ & & & & & & 1014.1 & 11.2 & $\mathrm{~A}^{\prime}$ & \\
\hline$v_{20}$ & 952.8 & 27.0 & $\mathbf{9 5 3}, 945$ & 953.8 & 28.1 & 956.1 & 22.2 & $\mathrm{~A}^{\prime}$ & $v_{\mathrm{g}}(\mathrm{CC})$ \\
\hline$v_{21}$ & $884.4, \mathbf{8 8 3 . 5}$ & 10.8 & 882,880 & 881.4 & 19.2 & 884.5 & 19.2 & $\mathrm{~A}^{\prime}$ & $\delta_{\mathrm{a}}($ ring $)$ \\
\hline$v_{22}$ & $697.4,695.6$ & 23.8 & 695 & 702.2 & 17.6 & 705.6 & 15.7 & $\mathrm{~A}^{\prime}$ & $v_{\mathrm{g}}(\mathrm{CC})+\delta_{\mathrm{b}}($ ring $)$ \\
\hline$v_{23}$ & 599.8 & 10.1 & 592 & 591.9 & 9.7 & 594.8 & 8.9 & $\mathrm{~A}^{\prime}$ & $v(C S)-v_{g}(C C)$ \\
\hline$v_{24}$ & 495.2, 492.4 & 8.9 & 498,491 & 495.9 & 7.3 & 498.1 & 6.4 & $\mathrm{~A}^{\prime}$ & $\delta(\mathrm{CCO})$ \\
\hline$v_{25}$ & & - & & 417.0 & 1.3 & 420.6 & 1.5 & $\mathrm{~A}^{\prime}$ & $\delta_{\mathrm{c}}($ ring $)$ \\
\hline$v_{26}$ & & - & & 334.9 & 6.6 & 336.4 & 7.5 & $\mathrm{~A}^{\prime}$ & $\delta_{\mathrm{d}}($ ring $)$ \\
\hline$v_{27}$ & & - & & 308.8 & 1.7 & 311.2 & 1.7 & $\mathrm{~A}^{\prime}$ & $\delta(\mathrm{CCS})$ \\
\hline
\end{tabular}




\begin{tabular}{|c|c|c|c|c|c|c|c|c|c|c|}
\hline \multirow[b]{2}{*}{$v_{28}$} & \multicolumn{4}{|c|}{ A" symmetry } & \multirow[b]{2}{*}{1007.7} & \multirow[b]{2}{*}{0.5} & \multirow[b]{2}{*}{1015.5} & \multirow[b]{2}{*}{0.3} & \multirow[b]{2}{*}{$\mathrm{A}^{\prime \prime}$} & \multirow[b]{2}{*}{$\gamma_{\mathrm{a}}(\mathrm{CH})$} \\
\hline & & - & - & & & & & & & \\
\hline$v_{29}$ & & - & - & & 992.0 & 0.05 & 1001.5 & 0.2 & $\mathrm{~A}^{\prime \prime}$ & $\gamma_{\mathrm{b}}(\mathrm{CH})$ \\
\hline$v_{30}$ & $925.4, \mathbf{9 2 3 . 1}$ & & 4.7 & 937 & 927.7 & 4.8 & 934.5 & 5.0 & $\mathrm{~A}^{\prime \prime}$ & $\gamma_{\mathrm{c}}(\mathrm{CH})$ \\
\hline$v_{31}$ & 854.1, 851.9 & & 0.7 & 866,864 & 856.2 & 0.2 & 874.8 & 6.4 & $\mathrm{~A}^{\prime \prime}$ & $\gamma_{\mathrm{d}}(\mathrm{CH})$ \\
\hline$v_{32}$ & $754.2, \mathbf{7 5 1 . 0}$ & & 17.9 & 757 & 770.4 & 32.1 & 825.2 & 26.2 & $\mathrm{~A}^{\prime \prime}$ & $\tau \mathrm{OH}$ \\
\hline$v_{33}$ & $734.8,732.6$ & & 71.4 & 738,723 & 734.2 & 71.8 & 748.2 & 12.5 & $\mathrm{~A}^{\prime \prime}$ & $\gamma_{\mathrm{e}}(\mathrm{CH})$ \\
\hline$v_{34}$ & 704.9 & & 6.6 & 701 & 701.5 & 8.3 & 713.6 & 62.3 & $\mathrm{~A}^{\prime \prime}$ & $\tau_{\mathrm{a}}($ ring $)$ \\
\hline$v_{35}$ & & - & - & 577 & 582.9 & 0.2 & 617.3 & 0.1 & $\mathrm{~A}^{\prime \prime}$ & $\tau_{\mathrm{b}}($ ring $)$ \\
\hline$v_{36}$ & & - & - & & 391.1 & 2.3 & 398.0 & 3.5 & $\mathrm{~A}^{\prime \prime}$ & $\tau_{c}(\operatorname{ring})$ \\
\hline$v_{37}$ & & - & - & & 339.2 & 0.8 & 347.9 & 1.9 & $\mathrm{~A}^{\prime \prime}$ & $\tau_{\mathrm{d}}($ ring $)$ \\
\hline$v_{38}$ & & - & - & & 166.8 & 0.02 & 171.1 & 0.003 & $\mathrm{~A}^{\prime \prime}$ & $\gamma(\mathrm{CO})-\gamma(\mathrm{CS})$ \\
\hline$v_{39}$ & & - & - & & 92.7 & 0.1 & 97.5 & 0.2 & $\mathrm{~A}^{\prime \prime}$ & $\gamma(\mathrm{CS})+\gamma(\mathrm{CO})$ \\
\hline
\end{tabular}

[a] Experimental intensities $(I)$ were normalized in such a way that the sum of integrated experimental intensities in the $1590-480 \mathrm{~cm}^{-1}$ frequency range would be equal to the sum of the corresponding harmonic infrared intensities $\left(A^{\text {th }}\right.$, in $\left.\mathrm{km} \mathrm{mol}^{-1}\right)$ of the two arrays $\left[\Sigma\left(v_{8}: v_{24}\right)+\Sigma\left(v_{30}: v_{34}\right)\right]$ computed at the B3LYP/6$311+\mathrm{G}(2 \mathrm{~d}, \mathrm{p})$ level of theory. For the split bands observed experimentally, the wavenumbers corresponding to the more intense component are shown in bold. The bands of the polycrystalline sample are designated as "crystal", for brevity.

[b] Computed harmonic wavenumbers were multiplied by 0.950 (above $2800 \mathrm{~cm}^{-1}$ ) or 0.980 (below $2000 \mathrm{~cm}^{-1}$ ) and are expressed in $\mathrm{cm}^{-1}$. Computed anharmonic wavenumbers and intensities are not scaled. The anharmonic section includes all fundamental modes, as well as the overtones and combination bands with computed anharmonic infrared intensities over $5 \mathrm{~km} \mathrm{~mol}^{-1}$.

[c] Approximate descriptions are based on visualization of normal vibrations (using the Chemcraft software ${ }^{\mathrm{S} 5}$ ), and also on the Vibrational Mode Automatic Relevance Determination (VMARD) analysis using Bayesian regression, as defined by Teixeira and Cordeiro. ${ }^{\mathrm{S}}$ The most prominent internal coordinates, obtained using the VMARD formalism, with weights above $5 \%$, are listed in Table S2.

[d] The band $v_{7}$ marked "ov." is predicted to appear near, and overlapped with the band due to monomeric $\mathrm{H}_{2} \mathrm{O}$ in an Ar matrix. ${ }^{\mathrm{S} 6}$ 
Table S4. The molecular vibrations of s-OH computed at the B3LYP/6-311+G(2d,p) level in harmonic approximation subjected to the VMARD (Vibrational Mode Automatic Relevance Determination) analysis using Bayesian regression.

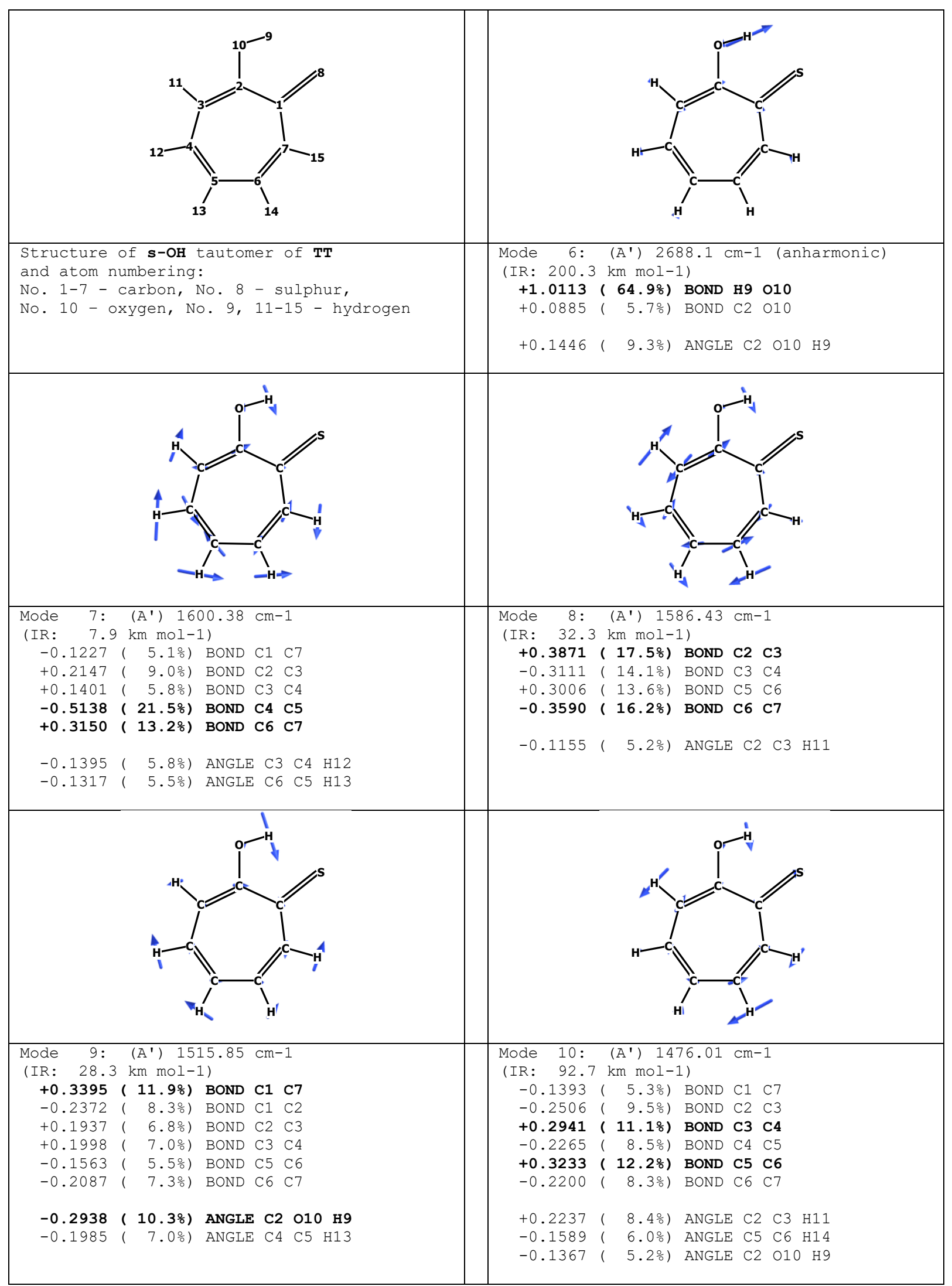




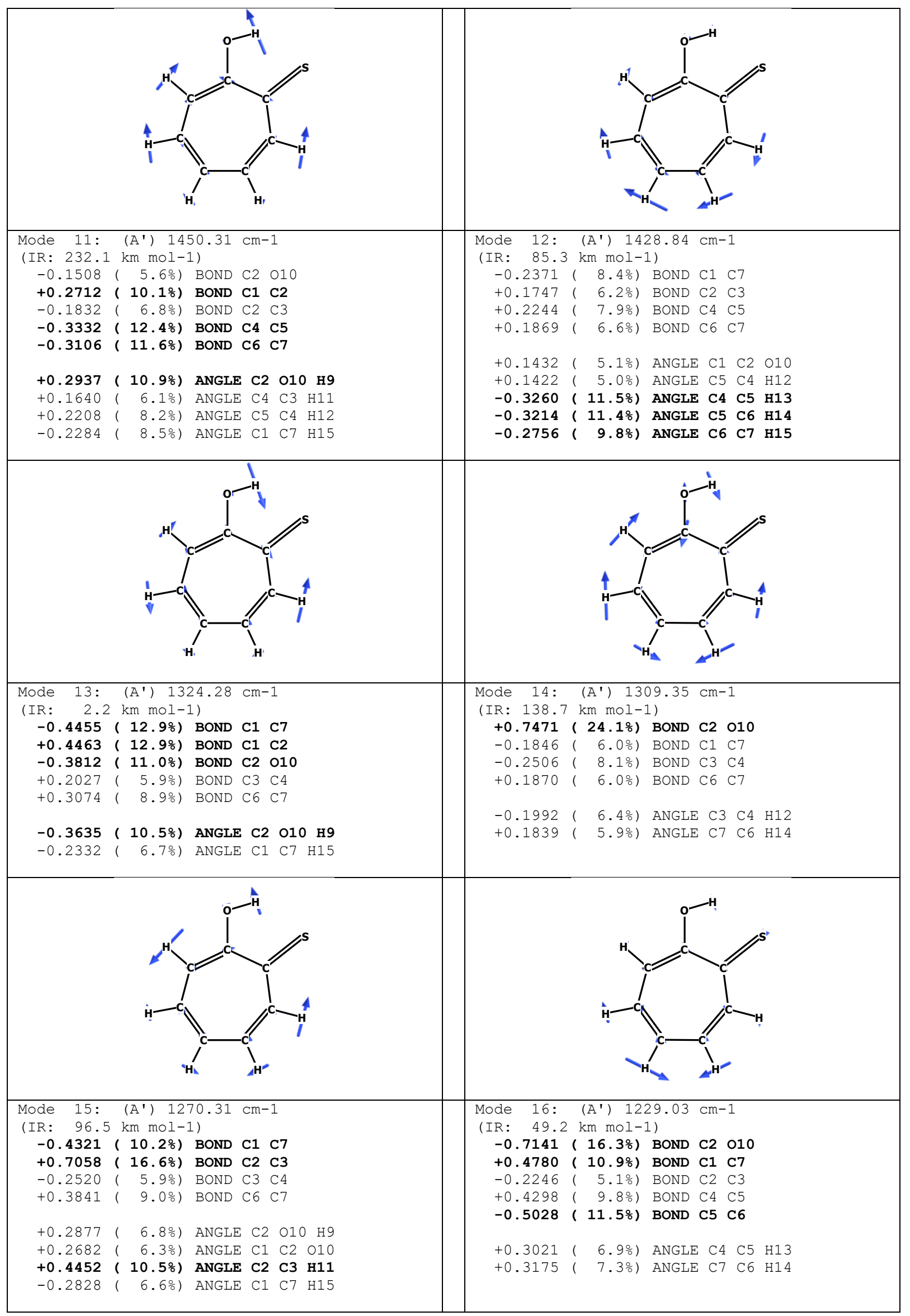




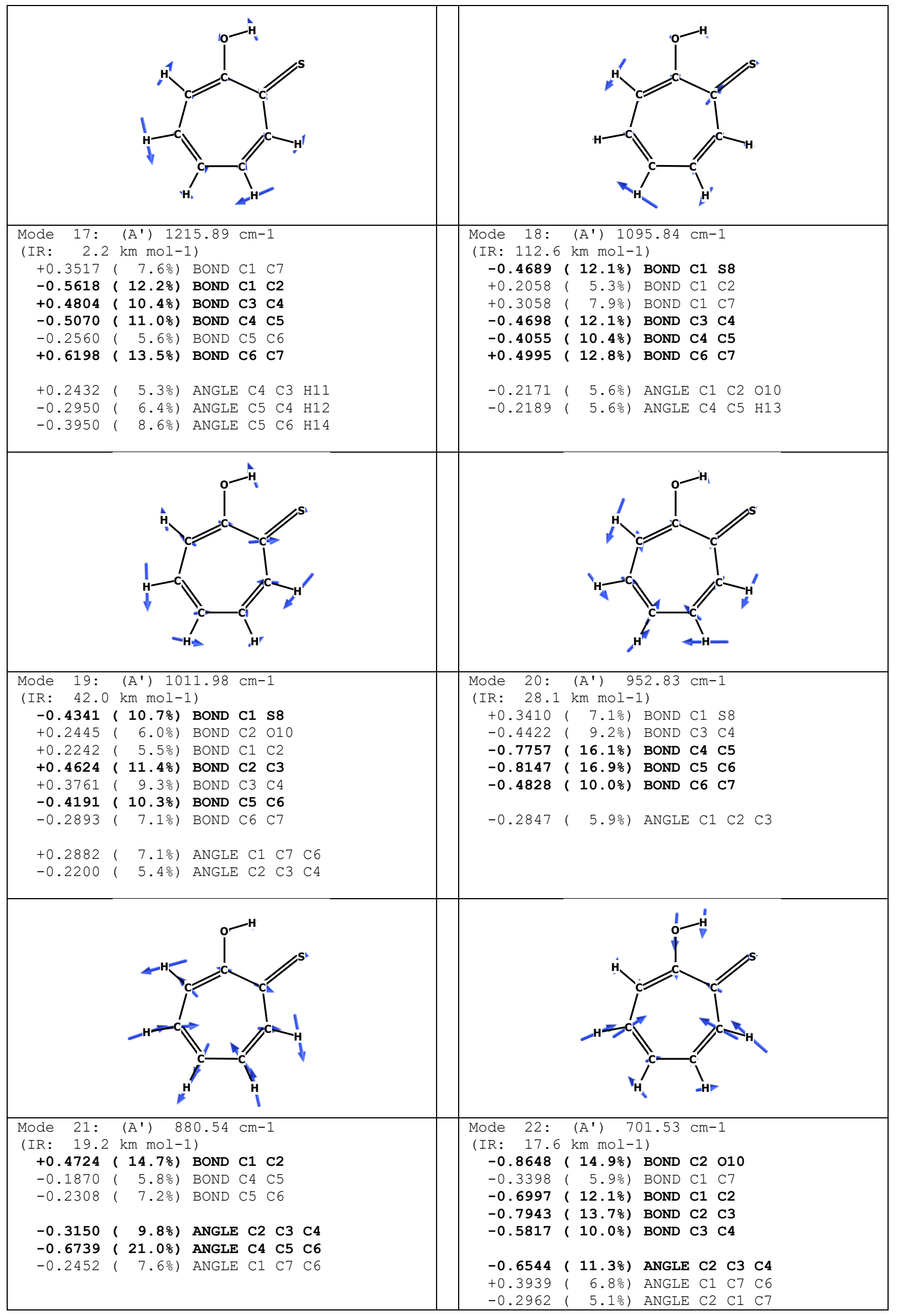




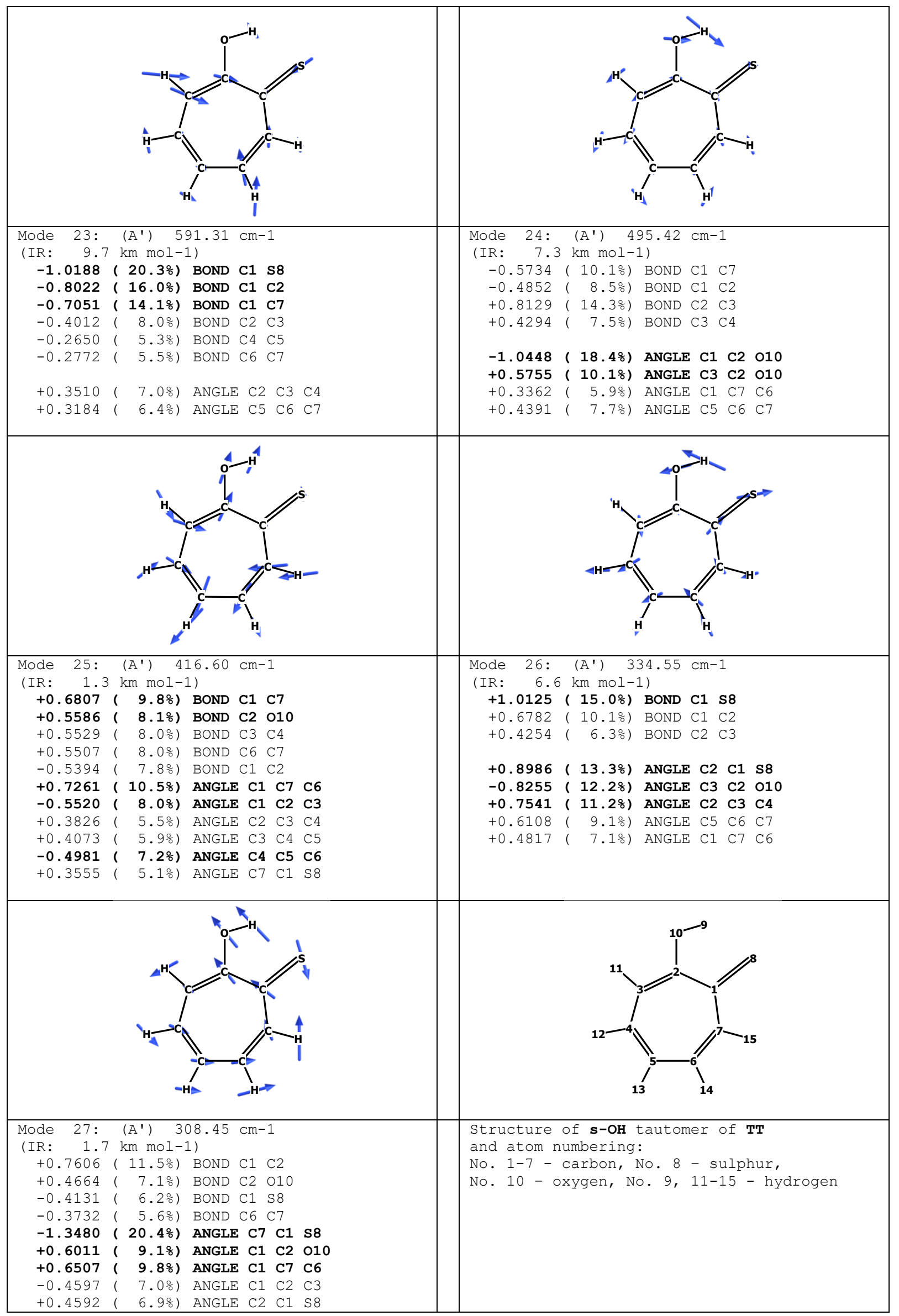




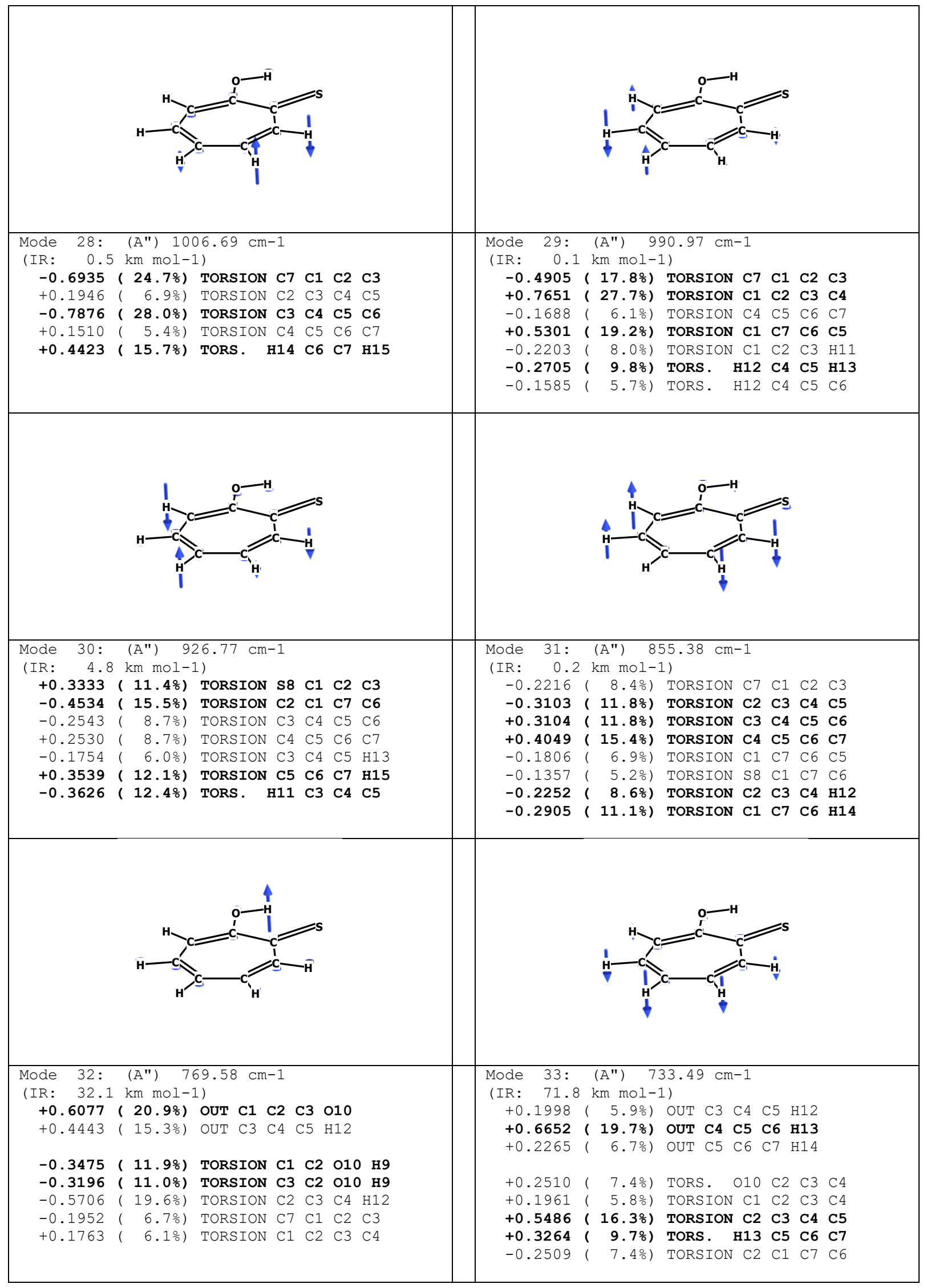




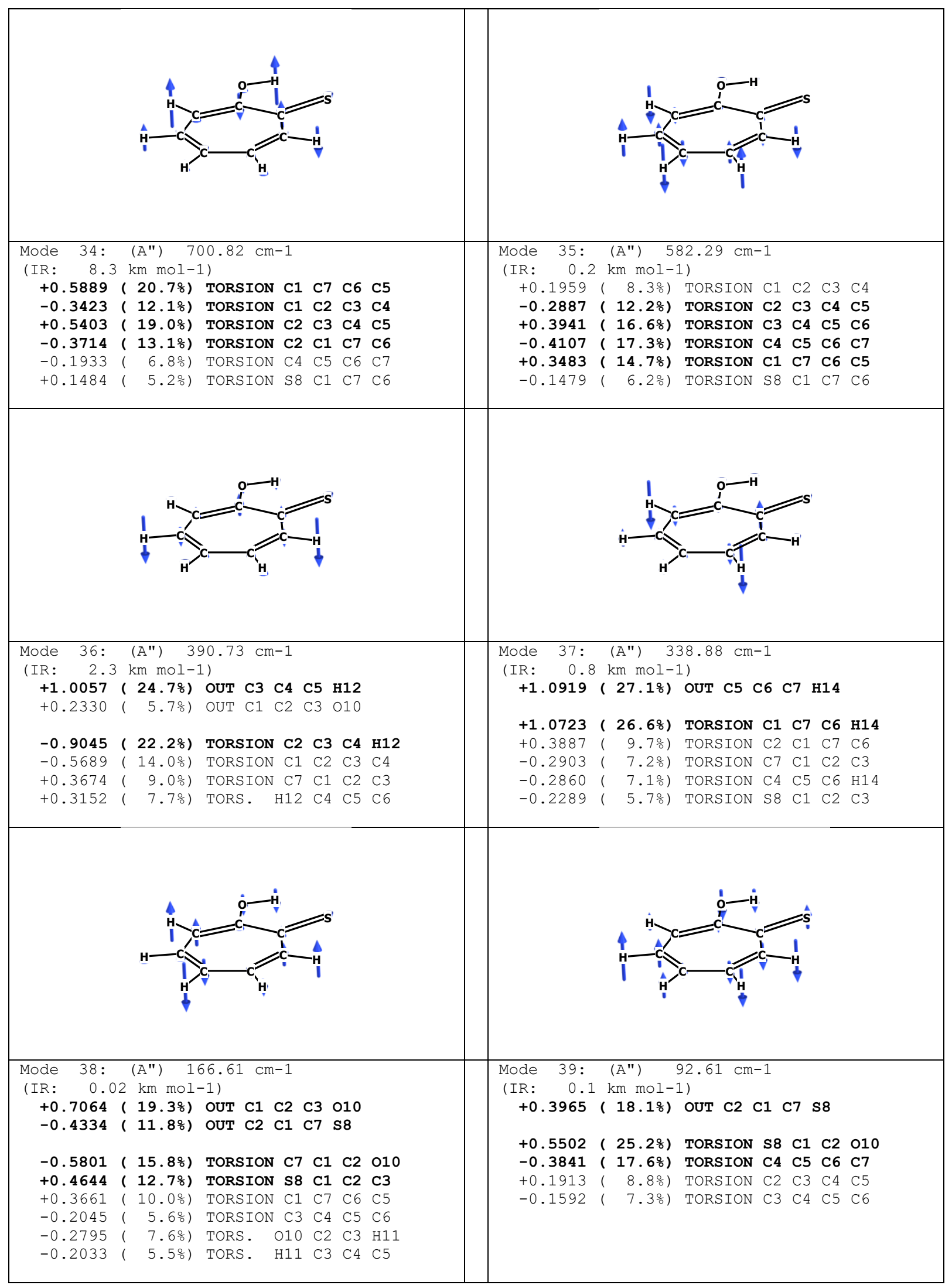


Table S5. Vibrational modes of the a-SH form of thiotropolone computed at the B3LYP/6$311+\mathrm{G}(2 \mathrm{~d}, \mathrm{p})$ level of theory in harmonic approximation, and vibrational assignment.

Mode Weight distribution $(\%)$ of most prominent internal coordinates ${ }^{[a, b]}$
Total Total Approximate

(\%) (\%) Description

Bond Angle

$\begin{array}{lll}97.6 & 2.4 & v_{a}(\mathrm{CH})\end{array}$

$96.4 \quad 3.6 \quad v_{b}(\mathrm{CH})$

$97.2 \quad 2.8 \quad v_{\mathrm{c}}(\mathrm{CH})$

$100.0 \quad 0.0 \quad v_{\mathrm{d}}(\mathrm{CH})$

$98.5 \quad 1.5 \quad v_{\mathrm{e}}(\mathrm{CH})$

$88.3 \quad 11.7 \quad v(\mathrm{SH})$

$\begin{array}{lll}76.6 & 23.4 & v_{a}(\mathrm{CC})\end{array}$

$80.0 \quad 20.0 \quad v(\mathrm{C}=\mathrm{O})$

$76.2 \quad 23.8 v_{b}(C C)-v_{c}(C C)$

$68.9 \quad 31.1 \quad v_{\mathrm{c}}(\mathrm{CC})+v_{\mathrm{b}}(\mathrm{CC})$ $-\delta_{\mathrm{a}}(\mathrm{CH})$

$64.4 \quad 35.6 \quad \delta_{\mathrm{a}}(\mathrm{CH})-v_{\mathrm{d}}(\mathrm{CC})$

$44.0 \quad 56.0 \quad \delta_{\mathrm{b}}(\mathrm{CH})$

(in-phase)

$53.0 \quad 47.0 \quad \delta_{\mathrm{c}}(\mathrm{CH})+v_{\mathrm{d}}(\mathrm{CC})$ ( $\delta$ syn)

$51.8 \quad 48.2 \quad \delta_{\mathrm{d}}(\mathrm{CH})-v_{\mathrm{e}}(\mathrm{CC})$ (Santi)

$57.0 \quad 43.0 \quad \delta_{\mathrm{e}}(\mathrm{CH})$ $(\delta \mathrm{C} 5 \mathrm{H}-\delta \mathrm{C} 6 \mathrm{H})$

$62.2 \quad 37.8 v_{\mathrm{e}}(\mathrm{CC})+\delta_{\mathrm{c}}(\mathrm{CH})$

$59.4 \quad 40.6 \quad v_{\mathrm{e}}(\mathrm{CC})+\delta_{\mathrm{d}}(\mathrm{CH})$

$58.4 \quad 41.6 \quad v_{\mathrm{f}}(\mathrm{CC})-\delta(\mathrm{SH})$

$60.6 \quad 39.4 \delta(\mathbf{S H})+v_{\mathrm{f}}(\mathrm{CC})$

$73.126 .9 \quad v_{\mathrm{g}}(\mathrm{CC})-$ $\delta_{\mathrm{a}}($ ring $)$

$42.3 \quad 57.7 \quad \delta_{b}($ ring $)$

$73.1 \quad 26.9 \quad v_{\mathrm{g}}(\mathrm{CC})+$ $\delta_{\mathrm{b}}$ (ring)

$74.5 \quad 25.5 v(\mathrm{CS})$

$47.0 \quad 53.0 \quad \delta(\mathrm{CCO})$

$49.1 \quad 50.9 \quad \delta_{\mathrm{c}}(\operatorname{ring})$

$47.1 \quad 52.9 \quad \delta_{\mathrm{d}}($ ring $)$

$37.3 \quad 62.7 \quad \delta(\mathrm{CCS})$ 


\section{A" symmetry}

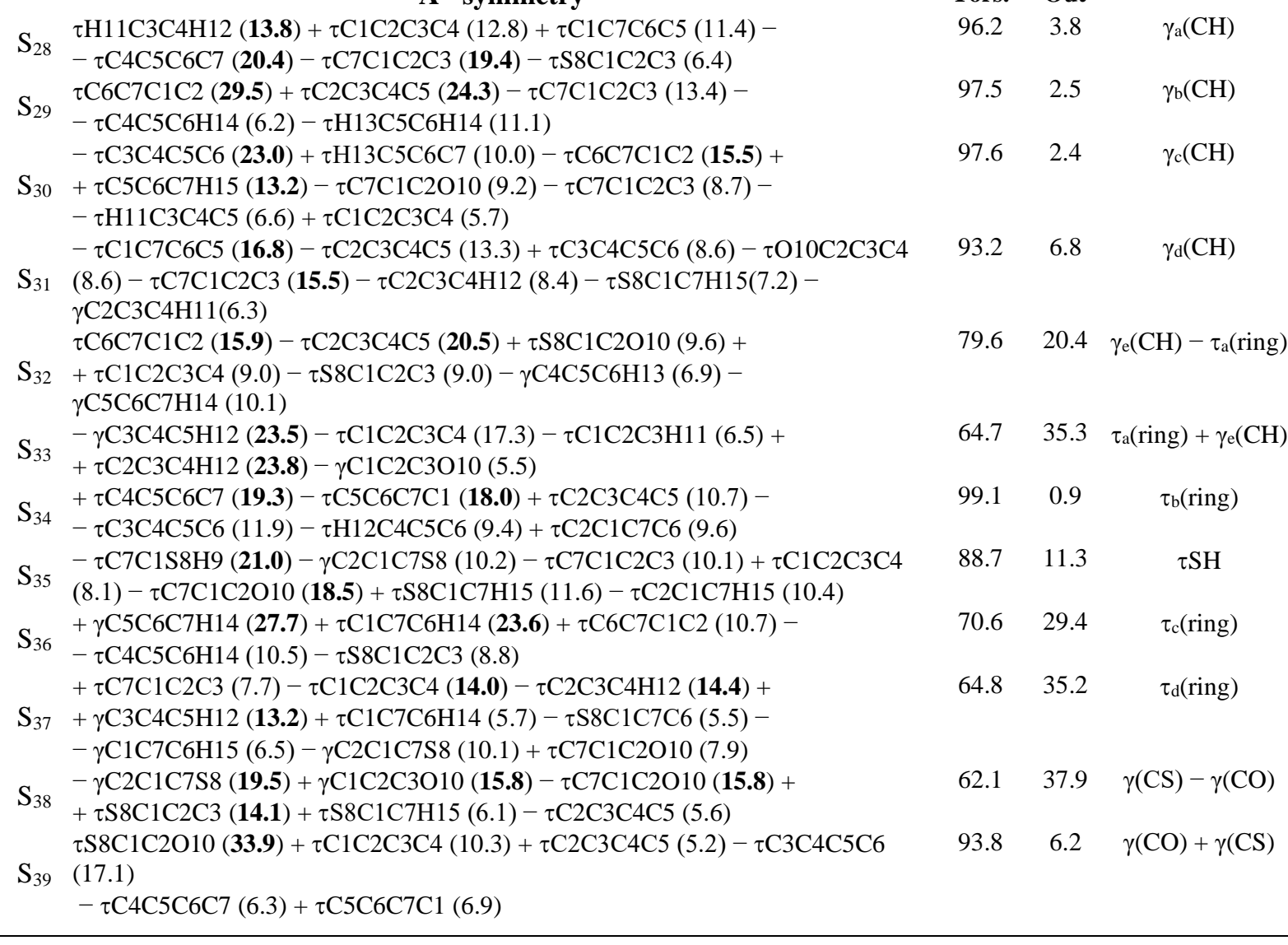

[a] See Scheme S2 for the atom numbering. Internal coordinates used in the analysis of vibrational modes are defomed automatically, as described in ref. S4.

[b] All contributions of most prominent internal coordinates, with weights of 5\% or higher, are included. The top contributions are shown in bold. Abbreviations: $v=$ stretching, $\delta=$ in-plane bending, $\gamma=$ out-of-plane bending, $\tau=$ torsion; $r_{i, j}$ is the distance between atoms $A_{i}$ and $A_{j} ; \beta_{i, j, k}$ is the angle between vectors $A_{k} A_{i}$ and $A_{k} A_{j} ; \tau_{i, j, k, l}$ is the dihedral angle between the plane defined by $A_{i}, A_{j}, A_{k}$ and the plane defined by $A_{j}, A_{k}$ and $A_{l}$ atoms; $\gamma_{i, j, k, l}$ is the angle between the vector $A_{k} A_{i}$ and the plane defined by atoms $A_{j}, A_{k}, A_{l}$. The symbols (+) and (-) denote in-phase and in-opposite-phase couplings between coordinates of different types.

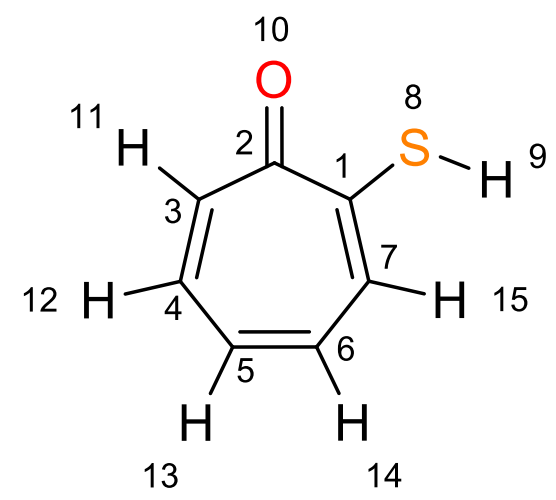

Scheme S2. Structure and the atom numbering of the a-SH form of thiotropolone. 
Table S6. Experimental IR spectra of a-SH form of TT isolated in an Ar matrix at $15 \mathrm{~K}$, and in neat solid TT crystals at $15 \mathrm{~K}$, compared with harmonic and anharmonic wavenumbers $\left(\tilde{v} / \mathrm{cm}^{-1}\right)$ and infrared intensities $\left(I / \mathrm{km} \mathrm{mol}^{-1}\right)$ computed at the B3LYP/6-311+G(2d,p) level of theory, and vibrational assignment.

\begin{tabular}{|c|c|c|c|c|c|c|c|c|c|}
\hline \multirow{2}{*}{$\begin{array}{l}\text { Mode } \\
\mathrm{N}^{\mathrm{o}}\end{array}$} & \multicolumn{2}{|c|}{ Ar matrix } & \multirow{2}{*}{${\frac{\text { Crystal }^{[\mathrm{a}]}}{\tilde{v}_{e x p}}}$} & \multicolumn{3}{|c|}{ Calc. $^{[b]}$} & & \multirow[t]{2}{*}{ Sym. } & \multirow{2}{*}{$\begin{array}{l}\text { Approximate } \\
\text { Description }^{[\mathrm{c}]}\end{array}$} \\
\hline & $\tilde{v}_{e x p}$ & $I_{e x p}$ & & $\tilde{v}_{\text {harm }}$ & $I_{\text {harm }}$ & $\tilde{v}_{\text {anhar }}$ & $I_{\text {anhar }}$ & & \\
\hline & $A^{\prime}$ symmetry & & & & & & & & \\
\hline$v_{7}+v_{10}$ & & & & & & 3112.7 & 5.5 & $\mathrm{~A}^{\prime}$ & \\
\hline$v_{9}+v_{10}$ & & & & & & 3041.9 & 6.4 & $\mathrm{~A}^{\prime}$ & \\
\hline$v_{1}$ & - & - & & 3014.8 & 11.1 & 3033.0 & 10.6 & $\mathrm{~A}^{\prime}$ & $v_{a}(\mathrm{CH})$ \\
\hline$v_{2}$ & - & - & & 3007.9 & 9.0 & 3052.4 & 10.2 & $\mathrm{~A}^{\prime}$ & $v_{b}(\mathrm{CH})$ \\
\hline$v_{3}$ & - & - & & 2997.6 & 8.2 & 3074.2 & 11.9 & $\mathrm{~A}^{\prime}$ & $v_{c}(\mathrm{CH})$ \\
\hline$v_{4}$ & - & - & & 2984.4 & 0.4 & 2997.9 & 4.8 & $\mathrm{~A}^{\prime}$ & $v_{\mathrm{d}}(\mathrm{CH})$ \\
\hline$v_{5}$ & - & - & & 2981.2 & 10.1 & 2960.6 & 0.05 & $\mathrm{~A}^{\prime}$ & $v_{e}(\mathrm{CH})$ \\
\hline$v_{6}$ & & & & 2638.8 & 7.3 & 2530.6 & 7.7 & $\mathrm{~A}^{\prime}$ & $v(\mathrm{SH})$ \\
\hline$v_{28}+v_{34}$ & & & & & & 1697.9 & 8.9 & $\mathrm{~A}^{\prime}$ & \\
\hline$v_{30}+v_{33}$ & & & 1664 & & & 1668.8 & 83.6 & $\mathrm{~A}^{\prime}$ & \\
\hline$v_{17}+v_{23}$ & & & 1639 & & & 1638.8 & 9.5 & $\mathrm{~A}^{\prime}$ & \\
\hline$v_{7}$ & 1627.7 & 79.3 & 1617 & 1626.7 & 60.0 & 1620.2 & 55.2 & $\mathrm{~A}^{\prime}$ & $v_{a}(C=C)$ \\
\hline $2 \times v_{32}$ & & & & & & 1612.2 & 5.5 & $\mathrm{~A}^{\prime}$ & \\
\hline$v_{30}+v_{34}$ & & & & & & 1607.4 & 6.8 & $\mathrm{~A}^{\prime}$ & \\
\hline$v_{14}+v_{26}$ & & & & & & 1602.9 & 12.9 & $\mathrm{~A}^{\prime}$ & \\
\hline FR (a) & $1602.3,1600.5$ & 67.0 & & & & & & & $\mathrm{FR}^{[\mathrm{d}]} \mathrm{v}_{8}$ with $\left[\mathrm{v}_{30}+\mathrm{v}_{33}\right]$ \\
\hline FR (b) & 1593.6 & 65.0 & & & & & & & $\mathrm{FR}^{[\mathrm{d}]} v_{8}$ with $\left[v_{21}+v_{22}\right]$ \\
\hline v8 (c) & 1586.7 & 134.1 & 1558,1552 & 1587.8 & 249.0 & 1590.7 & 50.9 & $\mathrm{~A}^{\prime}$ & $v(\mathrm{C}=\mathrm{O})$ \\
\hline$v_{17}+v_{24}$ & & & & & & 1587.6 & 25.9 & $\mathrm{~A}^{\prime}$ & \\
\hline$v_{15}+v_{26}$ & & & & & & 1585.8 & 9.8 & $\mathrm{~A}^{\prime}$ & \\
\hline$v_{21}+v_{22}$ & & & & & & 1585.5 & 11.6 & $\mathrm{~A}^{\prime}$ & \\
\hline \multirow[t]{2}{*}{$v_{9}(\mathbf{d})$} & 1565.3 shoulder & 11.0 & 1581 & 1586.1 & 19.7 & 1579.7 & 28.9 & $\mathrm{~A}^{\prime}$ & $v_{b}(C=C)$ \\
\hline & 1540.1 & 5.1 & & & & & & & \\
\hline$v_{10}$ & 1490.2, 1482.3 & 77.0 & $1487 \mathrm{sh}$. & 1484.1 & 40.1 & 1478.7 & 21.7 & $\mathrm{~A}^{\prime}$ & $v_{c}(\mathrm{C}=\mathrm{C})-\delta_{\mathrm{a}}(\mathrm{CH})$ \\
\hline$v_{11}$ & 1470.5 & 37.9 & 1467 & 1473.9 & 67.6 & 1468.5 & 60.1 & $\mathrm{~A}^{\prime}$ & $\delta_{\mathrm{a}}(\mathrm{CH})-v_{\mathrm{d}}(\mathrm{CC})$ \\
\hline$v_{12}$ & 1413.1 & 1.6 & 1423 & 1416.3 & 3.0 & 1414.5 & 2.0 & $\mathrm{~A}^{\prime}$ & $\delta_{\mathrm{b}}(\mathrm{CH})$ (in-phase) \\
\hline$v_{13}$ & 1393.5 & 14.8 & 1409,1390 & 1392.6 & 12.9 & 1388.4 & 10.9 & $\mathrm{~A}^{\prime}$ & $\delta_{\mathrm{c}}(\mathrm{CH})+v_{\mathrm{d}}(\mathrm{CC})(\delta \mathrm{syn})$ \\
\hline$v_{14}$ & 1277.0 & 7.0 & 1290 & 1269.5 & 18.5 & 1274.4 & 13.5 & $\mathrm{~A}^{\prime}$ & $\delta_{\mathrm{d}}(\mathrm{CH})-v_{\mathrm{e}}(\mathrm{CC})$ (Santi) \\
\hline$v_{15}$ & 1255.8 & 1.0 & 1256 & 1255.3 & 1.3 & 1262.4 & 1.9 & $\mathrm{~A}^{\prime}$ & $\delta_{\mathrm{e}}(\mathrm{CH})(\delta \mathrm{C} 5 \mathrm{H}-\delta \mathrm{C} 6 \mathrm{H})$ \\
\hline$v_{22}+v_{24}$ & & & & & & 1229.1 & 6.1 & $\mathrm{~A}^{\prime}$ & \\
\hline$v_{16}$ & 1219.2 & 6.3 & $\mathbf{1 2 2 4}, 1218$ & 1219.8 & 17.4 & 1213.4 & 7.4 & $\mathrm{~A}^{\prime}$ & $v_{\mathrm{e}}(\mathrm{CC})+\delta_{\mathrm{c}}(\mathrm{CH})$ \\
\hline$v_{34}+v_{37}$ & & & 1170 & & & 1151.5 & 8.1 & $\mathrm{~A}^{\prime}$ & \\
\hline$v_{17}$ & 1078.9 & 5.7 & 1078 & 1075.9 & 6.3 & 1079.3 & 0.9 & $\mathrm{~A}^{\prime}$ & $v_{\mathrm{e}}(\mathrm{CC})+\delta_{\mathrm{d}}(\mathrm{CH})$ \\
\hline$v_{18}$ & 1006.6 & 37.2 & 1001 & 1005.8 & 33.9 & 1004.7 & 29.7 & $\mathrm{~A}^{\prime}$ & $v_{f}(\mathrm{CC})-\delta(\mathrm{SH})$ \\
\hline$v_{19}$ & $\mathbf{9 3 5 . 3}, 939.8$ & 7.1 & 927 broad & 940.4 & 7.5 & 940.4 & 4.2 & $\mathrm{~A}^{\prime}$ & $\delta(\mathbf{S H})+v_{\mathrm{f}}(\mathrm{CC})$ \\
\hline$v_{20}$ & 917.7, 915.6 & 34.5 & $915 \mathrm{ov}$ & 915.9 & 43.2 & 919.4 & 38.2 & $\mathrm{~A}^{\prime}$ & $v_{\mathrm{g}}(\mathrm{CC})-\delta_{\mathrm{a}}($ ring $)$ \\
\hline$v_{21}$ & 875.7 & 12.7 & 872 & 872.1 & 22.2 & 874.0 & 15.7 & $\mathrm{~A}^{\prime}$ & $\delta_{b}($ ring $)$ \\
\hline$v_{22}$ & 717.6 & 6.2 & 743,730 & 706.4 & 10.0 & 713.6 & 5.7 & $\mathrm{~A}^{\prime}$ & $v_{\mathbf{g}}(\mathbf{C C})+\delta_{\mathrm{a}}($ ring $)$ \\
\hline$v_{23}$ & $\mathbf{5 6 3 . 5}, 561.8$ & 26.3 & 562 & 556.3 & 20.2 & 558.2 & 18.0 & $\mathrm{~A}^{\prime}$ & $v(\mathrm{CS})$ \\
\hline$v_{24}$ & 512.6 & 5.5 & 510 & 503.0 & 8.4 & 508.2 & 6.5 & $\mathrm{~A}^{\prime}$ & $\delta(\mathrm{CCO})$ \\
\hline$v_{25}$ & 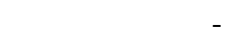 & - & & 407.4 & 4.5 & 412.7 & 3.1 & $\mathrm{~A}^{\prime}$ & $\delta_{\mathrm{c}}($ ring $)$ \\
\hline$v_{26}$ & - & - & & 320.2 & 0.8 & 323.8 & 0.9 & $\mathrm{~A}^{\prime}$ & $\delta_{\mathrm{d}}($ ring $)$ \\
\hline$v_{27}$ & - & - & & 237.0 & 5.2 & 238.7 & 4.7 & $\mathrm{~A}^{\prime}$ & $\delta(\mathrm{CCS})$ \\
\hline
\end{tabular}




\begin{tabular}{|c|c|c|c|c|c|c|c|c|c|}
\hline \multirow{2}{*}{$v_{28}$} & \multicolumn{3}{|l|}{ A" symmetry } & \multirow[b]{2}{*}{1008.0} & \multirow[b]{2}{*}{0.2} & \multirow[b]{2}{*}{1016.1} & \multirow[b]{2}{*}{0.3} & \multirow[b]{2}{*}{$\mathrm{A}^{\prime \prime}$} & \multirow[b]{2}{*}{$\gamma_{\mathrm{a}}(\mathrm{CH})$} \\
\hline & & - & & & & & & & \\
\hline$v_{29}$ & & - & & 983.1 & 0.03 & 1003.0 & 0.1 & A" & $\gamma_{\mathrm{b}}(\mathrm{CH})$ \\
\hline$v_{30}$ & 923.3, $925.4 \mathrm{sh}$ & 14.7 & $915 \mathrm{ov}$ & 920.4 & 6.5 & 928.2 & 4.4 & A" & $\gamma_{\mathrm{c}}(\mathrm{CH})$ (mainly $\left.\mathrm{H} 15\right)$ \\
\hline$v_{31}$ & 848.0, 849.3 & 5.6 & 857 & 848.3 & 4.5 & 919.7 & 12.6 & A" & $\gamma_{\mathrm{d}}(\mathrm{CH})$ \\
\hline V32 & 770.2 & 37.8 & 776 & 765.9 & 43.5 & 820.6 & 3.7 & A" & $\gamma_{\mathrm{e}}(\mathrm{CH})-\tau_{\mathrm{a}}($ ring $)$ \\
\hline$v_{33}$ & 673.0 & 19.2 & 680 & 671.8 & 18.8 & 696.5 & 44.4 & A" & $\tau_{\mathrm{a}}($ ring $)+\gamma_{\mathrm{e}}(\mathrm{CH})$ \\
\hline$v_{34}$ & & - & & 568.0 & 0.0003 & 676.7 & 0.3 & A" & $\tau_{\mathrm{b}}($ ring $)$ \\
\hline$v_{35}$ & & - & & 405.7 & 7.2 & 403.0 & 4.5 & $\mathrm{~A}^{\prime \prime}$ & $\tau \mathrm{SH}$ \\
\hline$v_{36}$ & & - & & 347.6 & 3.2 & 358.6 & 6.5 & A" & $\tau_{\mathrm{c}}($ ring $)$ \\
\hline$v_{37}$ & & - & & 325.2 & 0.6 & 438.6 & 0.1 & A" & $\tau_{\mathrm{d}}($ ring $)$ \\
\hline$v_{38}$ & & - & & 154.4 & 0.2 & 163.8 & 0.1 & A" & $\gamma(\mathrm{CS})-\gamma(\mathrm{CO})$ \\
\hline$v_{39}$ & & - & & 66.3 & 4.5 & 77.3 & 3.8 & A" & $\gamma(\mathrm{CO})+\gamma(\mathrm{CS})$ \\
\hline
\end{tabular}

[a] Experimental intensities $(I)$ were normalized in such a way that the sum of integrated experimental intensities in the $1630-480 \mathrm{~cm}^{-1}$ frequency range would be equal to the sum of the corresponding harmonic infrared intensities $\left(A^{\text {th }}\right.$, in $\left.\mathrm{km} \mathrm{mol}^{-1}\right)$ of the two arrays $\left[\Sigma\left(v_{7}: v_{24}\right)+\Sigma\left(v_{30}: v_{33}\right)\right]$ computed at the B3LYP/6$311+\mathrm{G}(2 \mathrm{~d}, \mathrm{p})$ level of theory. For the split bands observed experimentally, the wavenumbers corresponding to the more intense component are shown in bold. For the polycrystalline sample (for brevity designated as "crystal"), the wavenumbers shown in italic correspond to the bands of the a-SH form of TT, that do not spectrally overlap with the bands of the s-OH form.

[b] Computed harmonic wavenumbers were multiplied by 0.950 (above $2800 \mathrm{~cm}^{-1}$ ) or 0.980 (below $2000 \mathrm{~cm}^{-1}$ ) and are expressed in $\mathrm{cm}^{-1}$. Computed anharmonic wavenumbers and intensities are not scaled. The anharmonic section includes all fundamental modes, as well as the overtones and combination bands with computed anharmonic infrared intensities over $5 \mathrm{~km} \mathrm{~mol}^{-1}$.

[c] Approximate descriptions are based on visualization of normal vibrations (using the Chemcraft software ${ }^{\mathrm{S} 5}$ ), and also on the Vibrational Mode Automatic Relevance Determination (VMARD) analysis using Bayesian regression, as defined by Teixeira and Cordeiro. ${ }^{\mathrm{S} 4}$ The most prominent internal coordinates, obtained using the VMARD formalism, with weights above 5\%, are listed in Table S5.

[d] FR stands for Fermi-resonance. The suggested FR combinations (tentative) are based on the experimentally observed wavenumbers of the respective fundamental modes (for TT in Ar matrix). The sum of intensities of the experimental multiplet band in the $\mathbf{1 6 5 0 - 1 5 5 0} \mathbf{~ c m}^{-1}$ frequency range ( $T$ T in Ar matrix), where $v(C=O)$ and $v(\mathrm{C}=\mathrm{C})$ bands appear [marked as (a)-(d)], corresponds to the sum of theoretical intensities due to $v_{8}$ and $v_{9}$. 
Table S7. The molecular vibrations of a-SH computed at the B3LYP/6-311+G(2d,p) level in harmonic approximation subjected to the VMARD (Vibrational Mode Automatic Relevance Determination) analysis using Bayesian regression

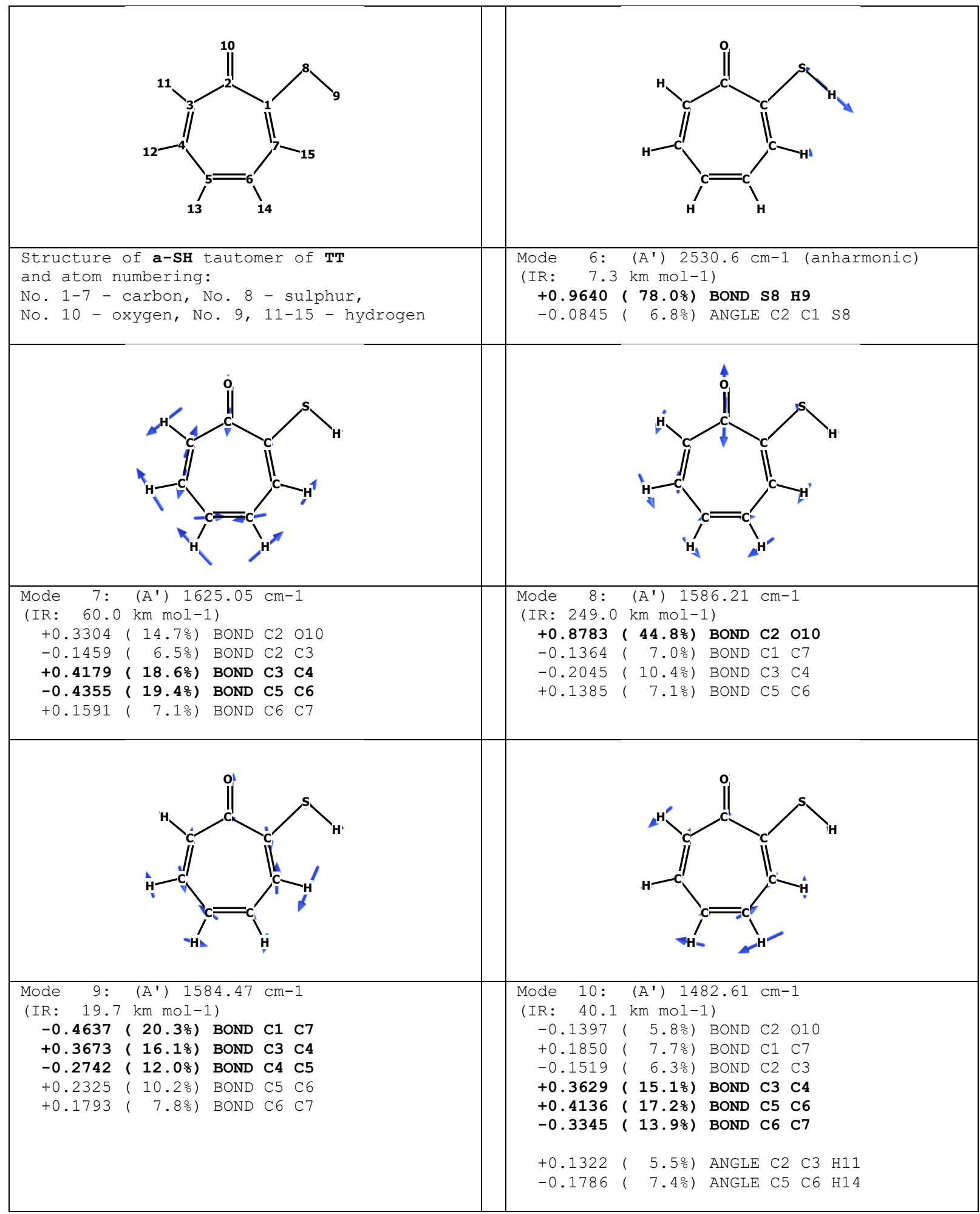




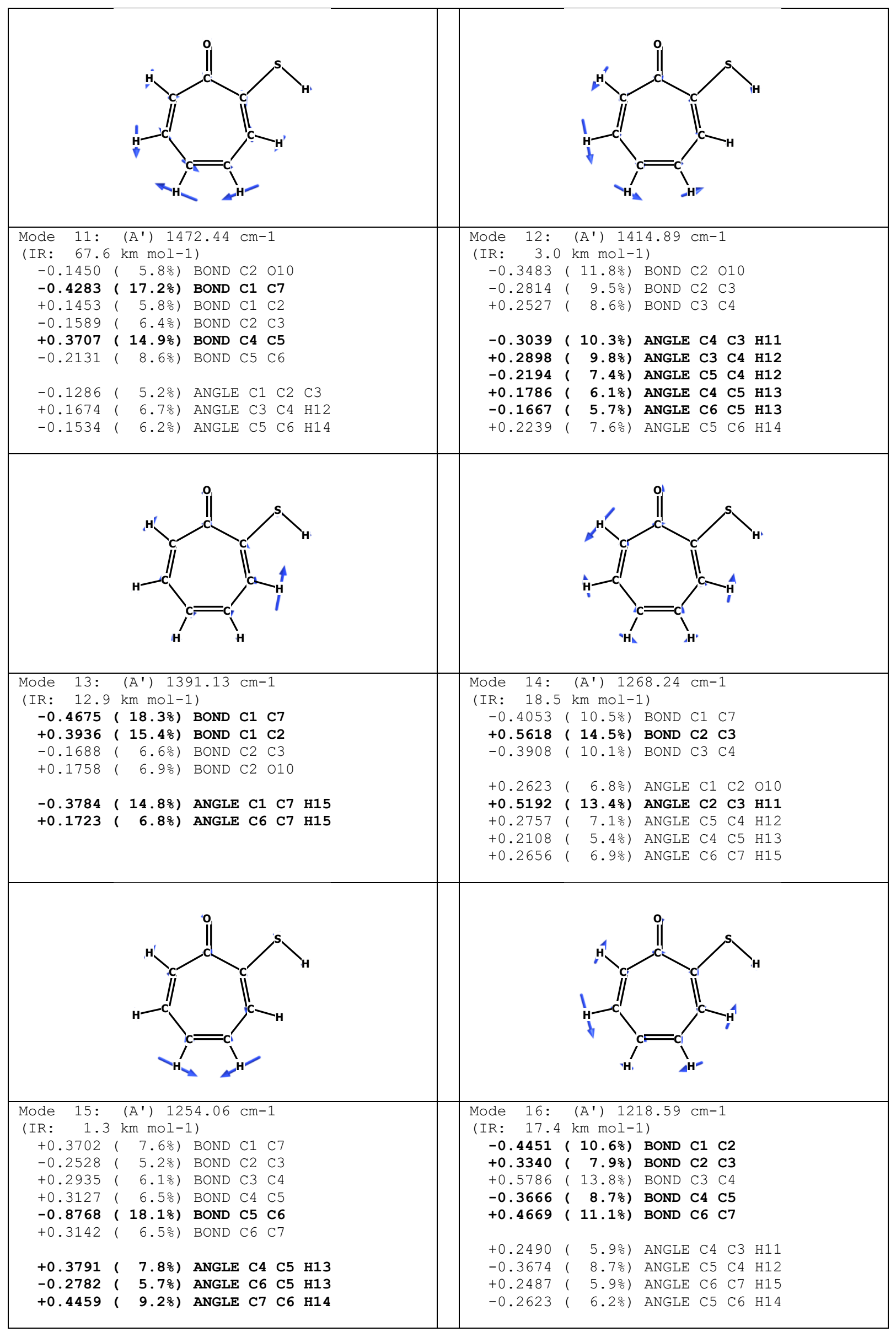




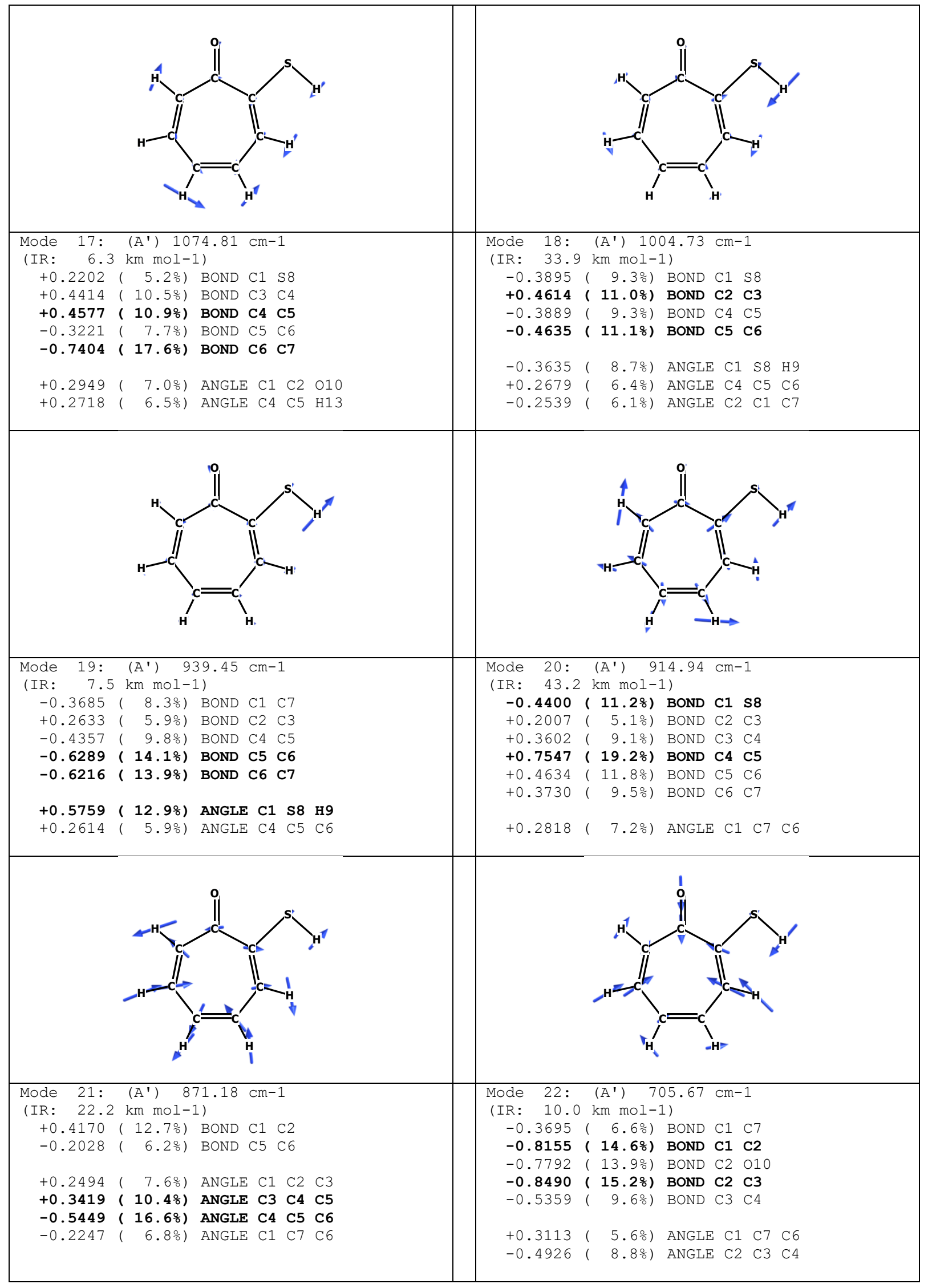




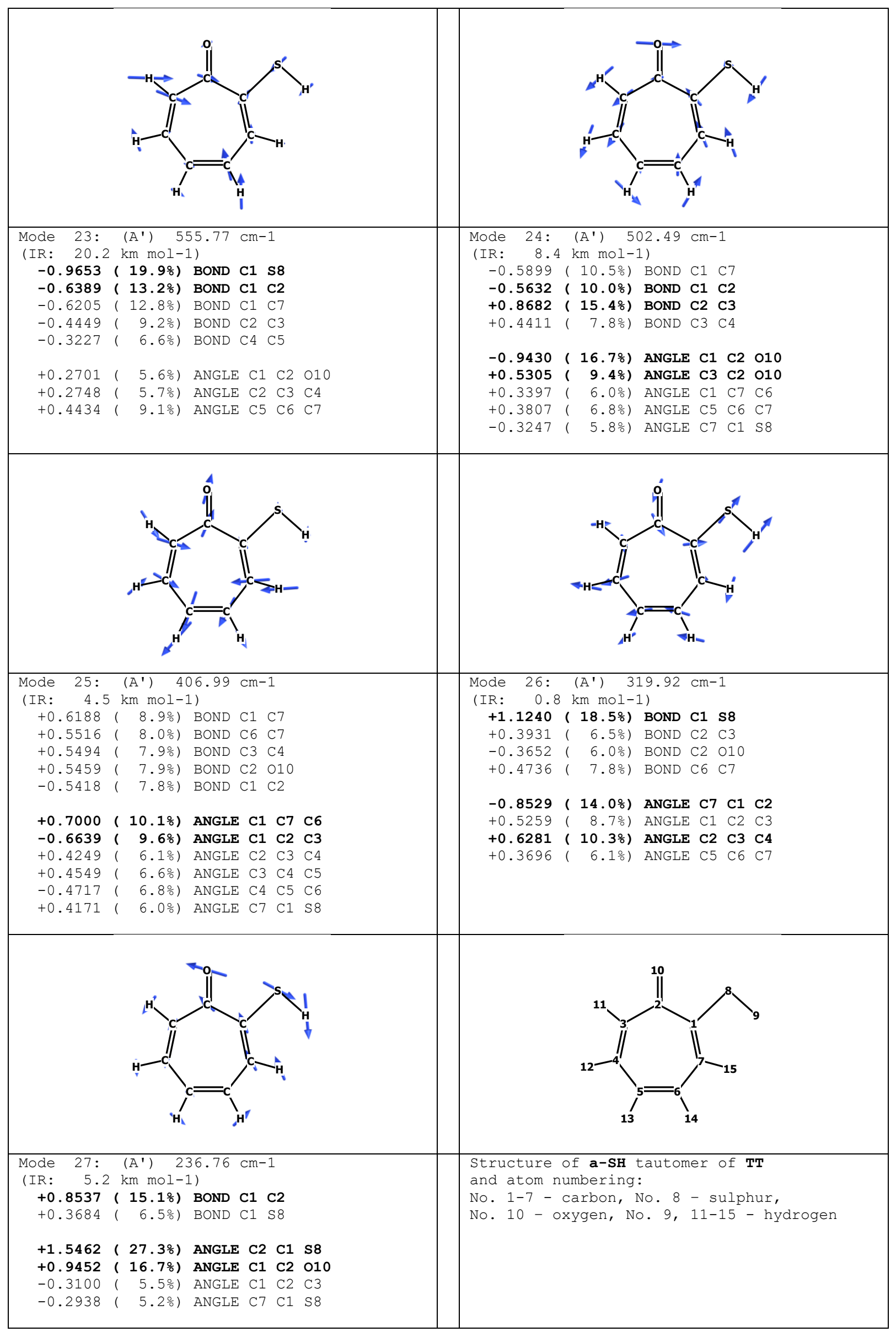




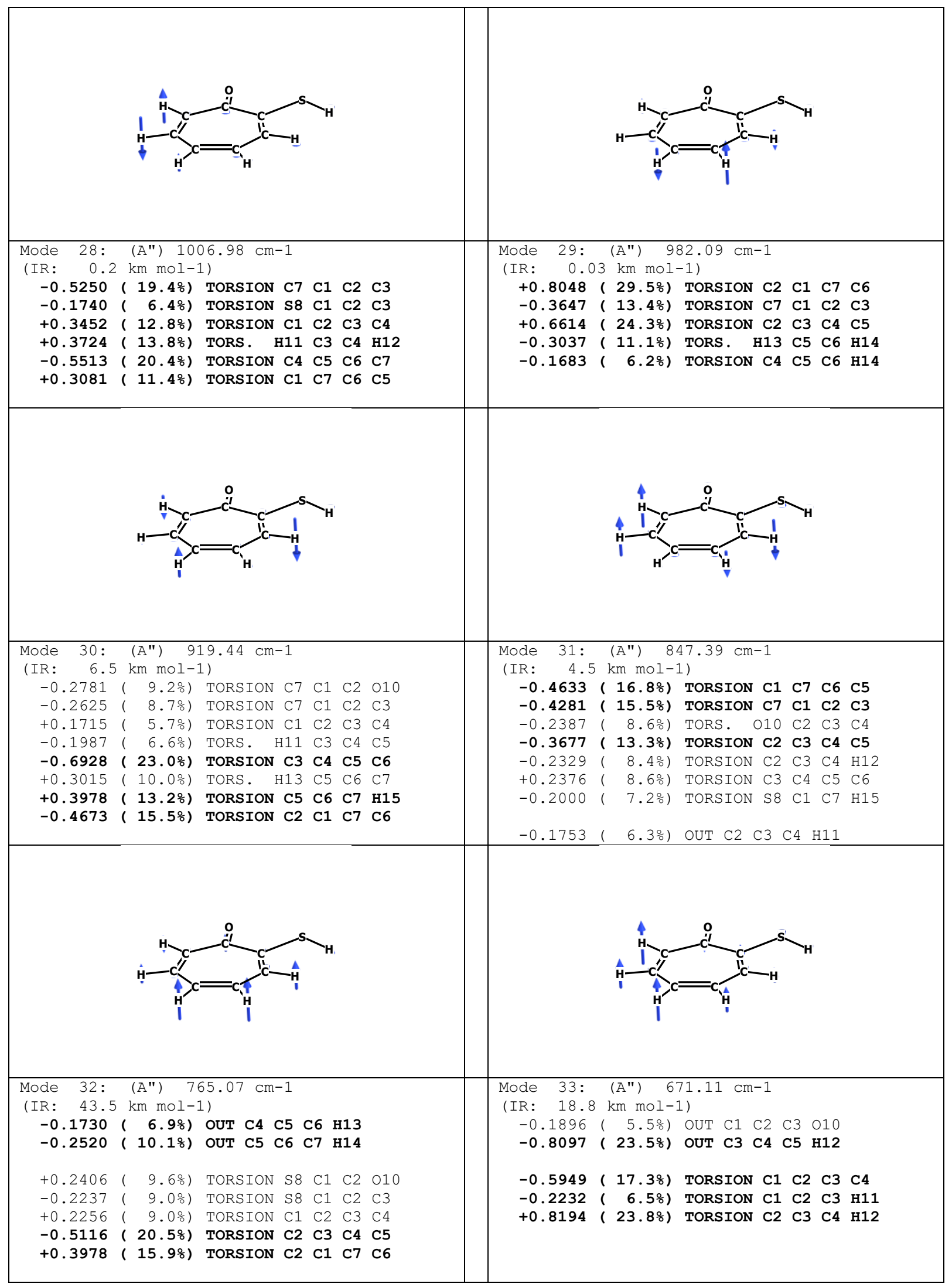




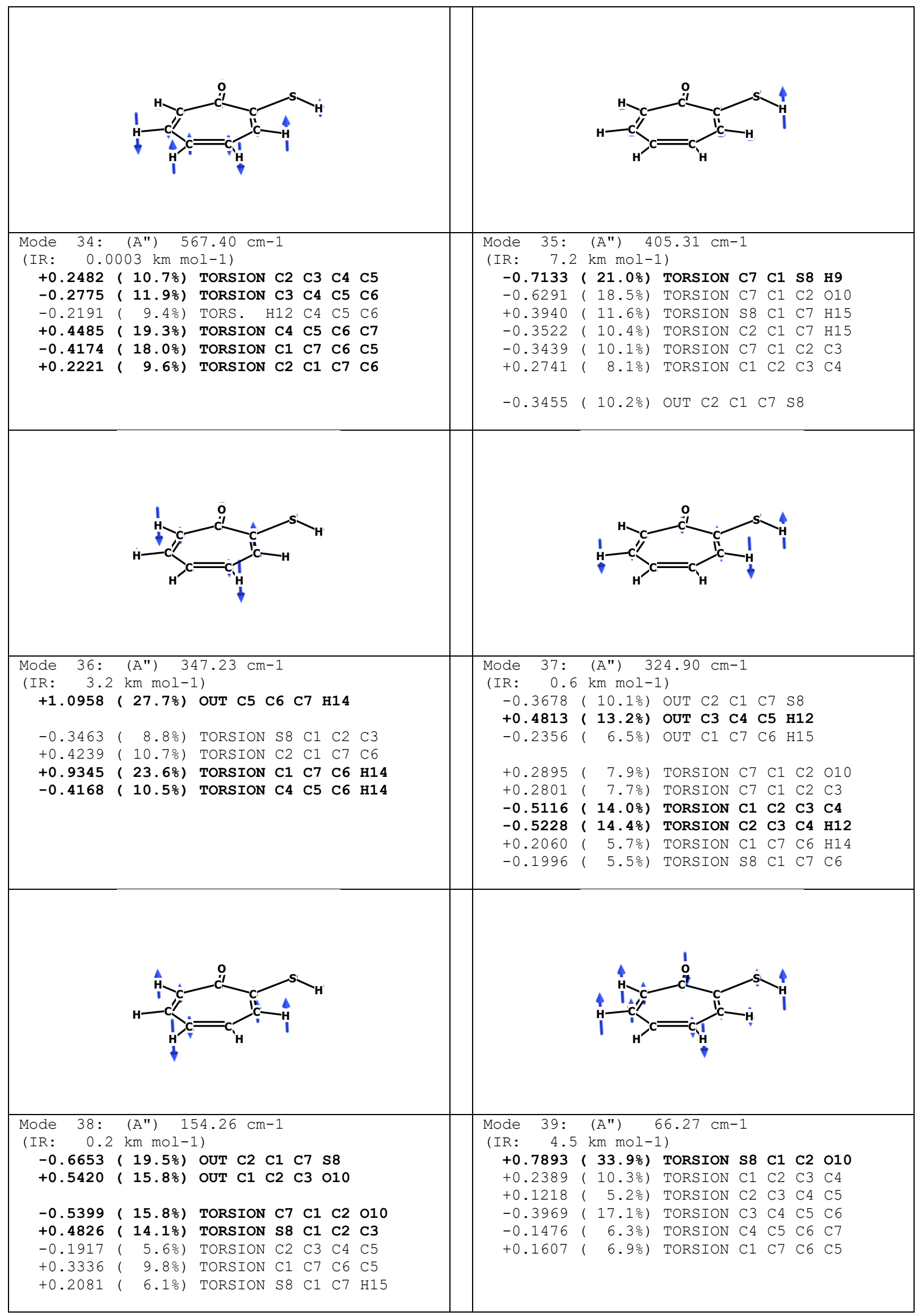


Table S8. Electronic energies ( $E_{\text {elec}}$, Hartree), zero-point energies (EZPE, Hartree), relative zero-point corrected energies $\left(\Delta \mathrm{E}_{0}, \mathrm{~kJ} \mathrm{~mol}^{-1}\right)$ calculated at $\mathrm{G} 4$ level of theory for the most relevant isomers of thiotropolone and for the tautomerization (taut) and rotamerization (rot) transition states (TS) involving these isomers.

\begin{tabular}{|c|c|c|c|c|}
\hline & s-OH & a-OH & s-SH & a-SH \\
\hline$E_{\text {elec }}$ & -743.535074 & -743.515105 & -743.528365 & -743.524997 \\
\hline $\mathrm{E}_{\mathrm{ZPE}}$ & 0.111162 & 0.111158 & 0.10791 & 0.108231 \\
\hline \multirow[t]{2}{*}{$\Delta \mathrm{E}_{0}$} & 0.0 & 52.4 & 9.1 & 18.8 \\
\hline & TS-(taut) & & TS-OH(rot) & TS-SH(rot) \\
\hline$E_{\text {elec }}$ & -743.521971 & & -743.508322 & -743.514029 \\
\hline $\mathrm{E}_{\mathrm{ZPE}}$ & 0.106059 & & 0.109745 & 0.106916 \\
\hline$\Delta \mathrm{E}_{0}$ & 21.0 & & 66.5 & 44.1 \\
\hline
\end{tabular}


Table S9. Optimized cartesian coordinates of the most relevant relevant isomers of thiotropolone calculated at G4 level of theory.

\begin{tabular}{lrrr}
\hline \multicolumn{4}{c}{$s$-OH } \\
\hline \multicolumn{4}{c}{ Coordinates (Angstroms) } \\
& $\mathrm{x}$ & $\mathrm{y}$ & $\mathrm{z}$ \\
\hline $\mathrm{C}$ & -0.693361 & -0.407379 & -0.000086 \\
$\mathrm{C}$ & -0.251035 & 0.989725 & 0.000005 \\
$\mathrm{C}$ & 1.043506 & 1.491263 & 0.000162 \\
$\mathrm{C}$ & 2.273707 & 0.827178 & 0.000275 \\
$\mathrm{C}$ & 2.530904 & -0.527892 & 0.000261 \\
$\mathrm{C}$ & 1.583953 & -1.562339 & 0.000125 \\
$\mathrm{C}$ & 0.205524 & -1.504353 & -0.000024 \\
$\mathrm{~S}$ & -2.367831 & -0.660430 & -0.000285 \\
$\mathrm{O}$ & -1.212742 & 1.895886 & -0.000070 \\
$\mathrm{H}$ & -2.064106 & 1.367915 & -0.000175 \\
$\mathrm{H}$ & 1.080567 & 2.576856 & 0.000200 \\
$\mathrm{H}$ & 3.143003 & 1.479633 & 0.000391 \\
$\mathrm{H}$ & 3.574132 & -0.828298 & 0.000365 \\
$\mathrm{H}$ & 1.995803 & -2.568728 & 0.000137 \\
$\mathrm{H}$ & -0.301359 & -2.464799 & -0.000111 \\
\hline
\end{tabular}

\begin{tabular}{lrrr}
\hline \multicolumn{4}{c}{$\boldsymbol{a}$-OH } \\
\hline & \multicolumn{4}{c}{ Coordinates (Angstroms) } \\
& $\mathrm{x}$ & $\mathrm{y}$ & $\mathrm{z}$ \\
\hline $\mathrm{C}$ & -0.738122 & -0.387091 & -0.000003 \\
$\mathrm{C}$ & -0.248209 & 0.995277 & -0.000006 \\
$\mathrm{C}$ & 1.053709 & 1.453854 & 0.000051 \\
$\mathrm{C}$ & 2.288396 & 0.771828 & 0.000116 \\
$\mathrm{C}$ & 2.519540 & -0.576333 & 0.000125 \\
$\mathrm{C}$ & 1.538335 & -1.593158 & 0.000069 \\
$\mathrm{C}$ & 0.171190 & -1.499533 & 0.000011 \\
$\mathrm{~S}$ & -2.383940 & -0.686137 & -0.000123 \\
$\mathrm{O}$ & -1.240801 & 1.899823 & -0.000070 \\
$\mathrm{H}$ & -0.868890 & 2.790326 & -0.000072 \\
$\mathrm{H}$ & 1.147179 & 2.540043 & 0.000048 \\
$\mathrm{H}$ & 3.162274 & 1.417614 & 0.000159 \\
$\mathrm{H}$ & 3.555126 & -0.902121 & 0.000174 \\
$\mathrm{H}$ & 1.925603 & -2.609224 & 0.000075 \\
$\mathrm{H}$ & -0.360870 & -2.446103 & -0.000028 \\
\hline
\end{tabular}

\begin{tabular}{lrrr}
\hline \multicolumn{4}{c}{$\boldsymbol{s}$-SH } \\
\hline \multicolumn{4}{c}{ Coordinates (Angstroms) } \\
& $\mathrm{x}$ & $\mathrm{y}$ & $\mathrm{z}$ \\
\hline $\mathrm{C}$ & -0.651375 & -0.376444 & -0.000001 \\
$\mathrm{C}$ & -0.273292 & 1.066906 & 0.000003 \\
$\mathrm{C}$ & 1.111590 & 1.496465 & 0.000005 \\
$\mathrm{C}$ & 2.287870 & 0.805113 & 0.000004 \\
$\mathrm{C}$ & 2.522625 & -0.590322 & 0.000000 \\
$\mathrm{C}$ & 1.580595 & -1.583034 & -0.000003 \\
$\mathrm{C}$ & 0.170899 & -1.477635 & -0.000004 \\
$\mathrm{~S}$ & -2.383007 & -0.638775 & -0.000003 \\
$\mathrm{O}$ & -1.162985 & 1.925383 & 0.000004 \\
$\mathrm{H}$ & -2.597706 & 0.707520 & -0.000002 \\
$\mathrm{H}$ & 1.176571 & 2.581121 & 0.000008 \\
$\mathrm{H}$ & 3.184647 & 1.420842 & 0.000006 \\
$\mathrm{H}$ & 3.563567 & -0.899202 & 0.000000 \\
$\mathrm{H}$ & 1.955159 & -2.603379 & -0.000006 \\
$\mathrm{H}$ & -0.343725 & -2.435848 & -0.000007 \\
\hline
\end{tabular}

\begin{tabular}{lrrr}
\hline \multicolumn{4}{c}{$\boldsymbol{a}$-SH } \\
\hline \multicolumn{4}{c}{ Coordinates (Angstroms) } \\
& $\mathrm{x}$ & $\mathrm{y}$ & $\mathrm{z}$ \\
\hline $\mathrm{C}$ & 0.640746 & -0.367799 & -0.000033 \\
$\mathrm{C}$ & 0.257039 & 1.064783 & -0.000005 \\
$\mathrm{C}$ & -1.129605 & 1.500580 & 0.000062 \\
$\mathrm{C}$ & -2.297795 & 0.800465 & 0.000111 \\
$\mathrm{C}$ & -2.516893 & -0.601953 & 0.000111 \\
$\mathrm{C}$ & -1.572162 & -1.589117 & 0.000061 \\
$\mathrm{C}$ & -0.157896 & -1.480592 & -0.000004 \\
$\mathrm{~S}$ & 2.401022 & -0.459025 & -0.000115 \\
$\mathrm{O}$ & 1.165691 & 1.896585 & -0.000036 \\
$\mathrm{H}$ & 2.457320 & -1.807913 & -0.000111 \\
$\mathrm{H}$ & -1.194843 & 2.585334 & 0.000074 \\
$\mathrm{H}$ & -3.202836 & 1.403956 & 0.000157 \\
$\mathrm{H}$ & -3.555584 & -0.918814 & 0.000157 \\
$\mathrm{H}$ & -1.942900 & -2.610872 & 0.000071 \\
$\mathrm{H}$ & 0.356358 & -2.438171 & -0.000034 \\
\hline
\end{tabular}


Table S10. Optimized cartesian coordinates calculated at G4 level of theory for the tautomerization (taut) and rotamerization (rot) transition states (TS) involving the most relevant isomers of thiotropolone (TT).

\begin{tabular}{crrr}
\hline \multicolumn{4}{c}{ TS $_{\text {rot-OH }}$} \\
\hline & \multicolumn{3}{c}{ Coordinates (Angstroms) } \\
& $\mathrm{x}$ & $\mathrm{y}$ & $\mathrm{z}$ \\
\hline $\mathrm{C}$ & -0.728534 & -0.389800 & -0.032848 \\
$\mathrm{C}$ & -0.251435 & 0.993671 & -0.058499 \\
$\mathrm{C}$ & 1.035532 & 1.464882 & 0.013769 \\
$\mathrm{C}$ & 2.280681 & 0.792923 & 0.105085 \\
$\mathrm{C}$ & 2.519852 & -0.549371 & 0.061367 \\
$\mathrm{C}$ & 1.552261 & -1.579104 & -0.071568 \\
$\mathrm{C}$ & 0.188880 & -1.501267 & -0.119401 \\
$\mathrm{~S}$ & -2.362733 & -0.711982 & 0.103640 \\
$\mathrm{O}$ & -1.229456 & 1.936013 & -0.205246 \\
$\mathrm{H}$ & -1.366620 & 2.425278 & 0.612210 \\
$\mathrm{H}$ & 1.097280 & 2.550297 & -0.006632 \\
$\mathrm{H}$ & 3.146027 & 1.443657 & 0.195004 \\
$\mathrm{H}$ & 3.555496 & -0.870835 & 0.121499 \\
$\mathrm{H}$ & 1.957543 & -2.586512 & -0.127588 \\
$\mathrm{H}$ & -0.333764 & -2.449878 & -0.198206 \\
\hline
\end{tabular}

\begin{tabular}{lrrr}
\hline \multicolumn{4}{c}{ TS $_{\text {rot }}$-SH } \\
\hline & \multicolumn{3}{c}{ Coordinates (Angstroms) } \\
& $\mathrm{x}$ & $\mathrm{y}$ & $\mathrm{z}$ \\
\hline $\mathrm{C}$ & 0.665419 & -0.303304 & -0.011551 \\
$\mathrm{C}$ & 0.210854 & 1.115392 & -0.017080 \\
$\mathrm{C}$ & -1.221210 & 1.458753 & 0.029556 \\
$\mathrm{C}$ & -2.348294 & 0.706790 & 0.036943 \\
$\mathrm{C}$ & -2.493210 & -0.713636 & 0.005287 \\
$\mathrm{C}$ & -1.501995 & -1.641944 & -0.024481 \\
$\mathrm{C}$ & -0.086056 & -1.440367 & -0.034864 \\
$\mathrm{~S}$ & 2.459367 & -0.517220 & -0.049827 \\
$\mathrm{O}$ & 1.018890 & 2.032824 & -0.050392 \\
$\mathrm{H}$ & 2.608083 & -0.746147 & 1.269301 \\
$\mathrm{H}$ & -1.342906 & 2.538468 & 0.060577 \\
$\mathrm{H}$ & -3.284241 & 1.259873 & 0.071127 \\
$\mathrm{H}$ & -3.515040 & -1.082719 & 0.007828 \\
$\mathrm{H}$ & -1.807578 & -2.684378 & -0.044892 \\
$\mathrm{H}$ & 0.487637 & -2.362271 & -0.066427 \\
\hline
\end{tabular}

\begin{tabular}{crrr}
\hline \multicolumn{4}{c}{ TS $_{\text {taut-OH-SH }}$} \\
\hline \multicolumn{4}{c}{ Coordinates (Angstroms) } \\
& $\mathrm{x}$ & $\mathrm{y}$ & $\mathrm{Z}$ \\
\hline $\mathrm{C}$ & -0.643785 & -0.434166 & -0.000097 \\
$\mathrm{C}$ & -0.289230 & 1.007964 & 0.000110 \\
$\mathrm{C}$ & 1.037801 & 1.519326 & 0.000261 \\
$\mathrm{C}$ & 2.250316 & 0.868708 & 0.000256 \\
$\mathrm{C}$ & 2.536713 & -0.504237 & 0.000099 \\
$\mathrm{C}$ & 1.630212 & -1.545126 & -0.000092 \\
$\mathrm{C}$ & 0.229248 & -1.510631 & -0.000178 \\
$\mathrm{~S}$ & -2.358286 & -0.633892 & -0.000233 \\
$\mathrm{O}$ & -1.260063 & 1.816785 & 0.000150 \\
$\mathrm{H}$ & -2.269264 & 0.873307 & -0.000035 \\
$\mathrm{H}$ & 1.061107 & 2.605176 & 0.000401 \\
$\mathrm{H}$ & 3.119459 & 1.522546 & 0.000396 \\
$\mathrm{H}$ & 3.588320 & -0.773984 & 0.000133 \\
$\mathrm{H}$ & 2.057153 & -2.545039 & -0.000192 \\
$\mathrm{H}$ & -0.251346 & -2.485046 & -0.000333 \\
\hline
\end{tabular}




\section{References}

S1. Giuliano, B. M.; Reva, I.; Lapinski, L.; Fausto, R. Infrared Spectra and UltravioletTunable Laser Induced Photochemistry of Matrix-Isolated Phenol and Phenol-d5. Journal of Chemical Physics, 2012, 136, 024505.

S2. Duarte, L.; Khriachtchev, L.; Fausto, R.; Reva, I. Photoisomerization of Azobenzenes Isolated in Cryogenic Matrices. Phys. Chem. Chem. Phys., 2016, 18, 16802-16811.

S3. Duarte, L.; Giuliano, B. M.; Reva, I.; Fausto, R. Tautomers and UV-Induced Photoisomerization of a Strongly Intramolecularly H-Bonded Aromatic Azo-Dye: 1-(Cyclopropyl)diazo-2-Naphthol. J. Phys. Chem. A, 2013, 117, 10671-10680.

S4. Teixeira, F.; Cordeiro, M. N. D. S. Improving vibrational mode interpretation using Bayesian regression. J. Chem. Theory Comput. 2019, 15, 456-470.

S5. Zhurko, G. A. Chemcraft - Graphical Program for Visualization of Quantum Chemistry Computations, Version 1.8; http://www.chemcraftprog.com, 2016.

S6. Michaut, X.; Vasserot, A.-M.; Abouaf-Marguin, L. Temperature and Time Effects on the Rovibrational Structure of Fundamentals of $\mathrm{H}_{2} \mathrm{O}$ Trapped in Solid Argon: Hindered Rotation and RTC Satellite. Vib. Spectrosc. 2004, 34, 83-93. 